\title{
ON REE'S SERIES OF SIMPLE GROUPS
}

\author{
BY \\ HAROLD N. WARD(1)
}

Introduction. The purpose of this paper is to obtain the character tables of the finite simple groups of Ree related to the Lie algebra $G_{2}$ (presented in [16], [17]) from certain basic properties of these groups. In the process we shall derive a number of additional properties of the Ree groups. We incorporate the basic properties as conditions in the following definition:

Definition. A finite group $G$ will be said to be of Ree type if it satisfies the following five conditions:

I. The 2-Sylow subgroups of $G$ are elementary Abelian of order 8 .

II. $G$ has no normal subgroup of index 2 .

III. For some element $J$ of order 2 (an "involution") in $G$, the centralizer $C_{G}(J)$ of $J$ in $G$ is the direct product of $\langle J\rangle$ and $L$ where $L$ is isomorphic to the linear fractional group $\operatorname{LF}(2, q)$.

Condition I implies that $q \equiv 4+e(\bmod 8)$ where $e= \pm 1$.

IV. If $\langle R\rangle$ denotes a cyclic subgroup of order $(q+e) / 2$ in $L$, then the normalizer $N_{G}\left(\left\langle R_{0}\right\rangle\right)$ of any subgroup $\left\langle R_{0}\right\rangle \neq\langle 1\rangle$ of $\langle R\rangle$ is contained in $C_{G}(J)$.

V. Let $J^{\prime}$ be an involution of $L$ and $S$ an element of $L$ of order $(q-e) / 4$ which centralizes $J^{\prime}$. Then an element of $G$ of order 3 which normalizes $\left\langle J, J^{\prime}\right\rangle$ does not centralize $S$.

We call $q$ the characteristic of $G$. The verification of these conditions is straightforward from the description of Ree's groups in [17].

The existence of elements $R, J^{\prime}$, and $S$ of IV and $\mathrm{V}$ is a consequence of the known structure of $\operatorname{LF}(2, q)$ which will be summarized in paragraph I-1. Moreover, there is an involution $J^{\prime \prime}$ of $L$ commuting with $J^{\prime}$ for which $J^{\prime \prime} S J^{\prime \prime}=S^{-1}$; the centralizer of $J^{\prime}$ in $L$ is $\left\langle J^{\prime}, J^{\prime \prime}, S\right\rangle$. Thus the centralizer $C_{G}\left(\left\langle J, J^{\prime}\right\rangle\right)$ is $\left\langle J, J^{\prime}, J^{\prime \prime}, S\right\rangle$. Also, there is an element of order 3 in $L$ normalizing $\left\langle J^{\prime}, J^{\prime \prime}\right\rangle$ but not centralizing it.

Received by the editors September 21, 1964.

(1) This paper is essentially the author's 1962 Harvard dissertation with some simplification augmentation, and correction. The dissertation was written under the direction of Professor Richard Brauer to whom the author is extremely grateful for guidance and encouragement. In addition, the author wishes to thank the referee for his painstaking examination and very helpful commentary. The original research and the revisions were all supported by the National Science Foundation. 
Let $P_{2}$ be a 2-Sylow subgroup of $G$. Since $P_{2}$ is Abelian, the transfer of $G$ into $P_{2}$ has as its image the intersection of $P_{2}$ with the center of $N_{G}\left(P_{2}\right)[2, \mathrm{p}$. 173]. The automorphism group of an elmentary Abelian group of order 8 has order 168 , so that $\left[N_{G}\left(P_{2}\right): C_{G}\left(P_{2}\right)\right]$ divides 21 . From the above, 3 divides this index. But if the index is just 3, $N_{G}\left(P_{2}\right)$ has a 2-element in its center and the kernel of the transfer is proper, violating condition II. Hence the index is 21 and we conclude that all the members of $P_{2-}\langle 1\rangle$ are conjugate, and that any 4-group of $P_{2}$ is normalized but not centralized by an element of order 3 . Thus all involutions in $G$ are conjugate and an element of order 3 in condition V really exists.

The element $S$ of condition $\mathrm{V}$ cannot be the identity, so that the values 3 and 5 for $q$ are excluded. For $5<q<27$, the possible orders of $\langle S\rangle$ are (in light of III) 3 and 5; but cyclic groups of these orders do not possess automorphisms of order 3 . Thus in fact $q \geqq 27$.

The object of this paper is to prove the following theorem:

THEOREM. Let $G$ be a group of Ree type of characteristic $q$. Then the following hold:

(1) $G$ is simple of order $q^{3}(q-1)\left(q^{3}+1\right)$ where $q=3^{2 k+1}, k \geqq 1$.

(2) If $P$ is a 3-Sylow subgroup of $G, P$ has order $q^{3}$ and is disjoint from its conjugates. Its center $Z(P)$ is elementary Abelian of order $q, P$ is of class 3 , and $P$ contains a normal elementary Abelian subgroup $P_{1}$ of order $q^{2}$ containing $Z(P)$ which is both the derived group and the Frattini subgroup of $P$. The members of $P-P_{1}$ have order 9 , their cubes forming $Z(P)-\langle 1\rangle$.

(3) The normalizer $N(P)=P W$ where $W$ is cyclic of order $q-1$. If $J$ is the involution of $W, C_{P}(J)=C_{P_{1}}(J)$ is elementary Abelian of order $q$ and $C_{P}(J) \cap Z(P)=\langle 1\rangle$. If $R$ is an element of $W$ of $($ odd $)$ order $(q-1) / 2$, then $C_{P}\left(R^{a}\right)=\langle 1\rangle$ for all $R^{a} \neq 1$.

(4) $q^{2}-q+1=(q+1+3 m)(q+1-3 m)$, where $m=3^{k}$. G possesses Abelian Hall subgroups $M^{+}$and $M^{-}$of orders $q+1+3 m$ and $q+1-3 m$, respectively. $M^{+}$and $M^{-}$are respectively the centralizers of their nonidentity elements and are each disjoint from their conjugates. Furthermore, $N\left(M^{+}\right)=M^{+} W^{+}$ and $N\left(M^{-}\right)=M^{-} W^{-}$where $W^{+}$and $W^{-}$are cyclic of order $6 . W^{+}$and $W^{-}$ induce regular groups of automorphisms of $M^{+}$and $M^{-}$, respectively.

(5) The permutation representation of $G$ on the right cosets of $N(P)$ represents $G$ faithfully as a doubly transitive permutation group in such a way that the subgroup fixing three letters has order 2.

(6) The character table of $G$ is uniquely determined up to some values of certain "exceptional characters;" the table appears at the end of Chapter V.

(7) The decompositions of the various 2-blocks of $G$ are uniquely determined (and appear at the end of Chapter I).

The present paper is meant to fit into the general program of the characteri- 
zation of the known simple groups. Since from the result of Feit and Thompson noncyclic simple groups are of even order, the most natural approach to this problem is by a study of the structure of the group relating to its 2-Sylow subgroup and elements of even order.

More particularly, the material presented here bears on the general problem of characterizing groups with Abelian 2-Sylow subgroups. J. H. Walter has worked on showing that such simple groups, assuming their proper subgroups are known, must satisfy conditions I-III for groups of Ree type. Thompson and Janko in turn have shown that conditions IV and V follow from I-III provided $q>5$. Thus in the program of classifying groups with Abelian 2-Sylow subgroups, it must be shown that a group of Ree type really is one of Ree's groups. (The exceptional case $q=5$ (with condition $\mathrm{V}$ waived) corresponds to Janko's new simple group.)

One approach is by the study of permutation groups. Doubly transitive groups in which only the identity fixes three letters have been classified by Zassenhaus, Feit, Suzuki, and Ito. In addition, Suzuki has classified doubly transitive groups which are similar in nature to the Ree groups but act on an odd number of letters [20]. As an indication of how close the connection to permutation groups is, Ree $[18]$ has shown that a doubly transitive group on an even number $(\geqq 4)$ of letters such that the subgroup fixing two letters has exactly one nonidentity element fixing at least three letters and such that each involution fixes at least three letters is indeed of Ree type. (These properties of groups of Ree type follow readily from the discussion of Chapter III.)

The two papers of Brauer, [2] and [3], are referred to as BI and BII, and the numbering of these papers is followed.

\section{Chapter I. The Principal 2-Block of $G$ and Two Families of ExCEPTIONAL Characters}

The main result of this chapter is that the principal 2-block of $G$ has eight ordinary characters. Formulas of Brauer then lead to the degrees of these characters and the order of the group. These formulas are the main tool of the derivation, for they give number-theoretic requirements on $G$ which are very stringent. In preparation for these considerations, two families of exceptional characters are obtained from condition IV on $G$. Likewise, information about the 2-blocks of defects 1 and 2 is produced.

1. The following remarks describe the nonidentity conjugate classes of $L F(2, q)$ : There are two elements $R$ and $S_{0}$ of orders $\frac{1}{2}(q+e)$ and $\frac{1}{2}(q-e)$, respectively, each conjugate to its inverse but to no other power. There is a subgroup $\left\{T(x) \mid x \in F_{q}\right\}, F_{q}$ the field of $q$ elements, isomorphic to the additive group of $F_{q}$ by $x \rightarrow T(x) . T(x)$ and $T(y)$ are conjugate iff $x y$ is a square in $F_{q}$, for $x, y \neq 0$. Let $T$ and $T^{\prime}$ be members of this subgroup representing the two (nonidentity) classes so obtained. Let $S_{0}^{2}=S$ and $S_{0}^{(q-e) / 4}=J^{\prime}$ (an involution). The orders 
of the centralizers in $L F(2, q)$ are $\frac{1}{2}(q+e)$ for $R^{a} \neq 1 ; \frac{1}{2}(q-e)$ for $S_{0}^{b} \neq 1, J^{\prime}$; $q-e$ for $J^{\prime} ; q$ for $T(x) \neq 1$. The order of $L F(2, q)$ is $\frac{1}{2} q\left(q^{2}-1\right)$. (For all this see [11].)

2. The character table for $L F(2, q)$ is in Table 1 (from [14]). Here $r(\neq 1)$ is any $\frac{1}{2}(q+e)$ th root of unity and $s(\neq \pm 1)$ any $\frac{1}{2}(q-e)$ th root of unity; $r$ and $r^{-1}$ give the same character, as do $s$ and $s^{-1}$. There are $\frac{1}{4}(q+e-2) \theta_{r}$ 's and $\frac{1}{4}(q-e-4) \theta_{s}$ 's.

TABLE 1

\begin{tabular}{|c|c|c|c|c|c|}
\hline Value on & 1 & $T$ & $T^{\prime}$ & $R^{a}$ & $S_{0}^{b}$ \\
\hline Character & & & & & \\
\hline$\theta_{1}$ & 1 & 1 & 1 & 1 & 1 \\
\hline$\theta_{2}$ & $\frac{1}{2}(q+e)$ & $\frac{1}{2}\left(e+(e q)^{1 / 2}\right)$ & $\frac{1}{2}\left(e-(e q)^{1 / 2}\right)$ & 0 & $e(-1)^{b}$ \\
\hline$\theta_{3}$ & $\frac{1}{2}(q+e)$ & $\frac{1}{2}\left(e-(e q)^{1 / 2}\right)$ & $\frac{1}{2}\left(e+(e q)^{1 / 2}\right)$ & 0 & $e(-1)^{b}$ \\
\hline$\theta_{4}$ & $q$ & 0 & 0 & $-e$ & $e$ \\
\hline$\theta_{r}$ & $q-e$ & $-e$ & $-e$ & $(-e)\left(r^{a}+r^{-a}\right)$ & 0 \\
\hline$\theta_{s}$ & $q+e$ & $e$ & $e$ & 0 & $e\left(s^{b}+s^{-b}\right)$ \\
\hline
\end{tabular}

3. The modular characters for the prime 2 of $L F(2, q)$ are as follows (facts used but not cited are in $\mathrm{BI}$ ): the number of modular characters is the number of 2-regular classes, and that is $2+(3 q+e) / 8$. The $\theta_{r}$ are of defect 0 and thus are the sole members of their blocks and provide modular characters when restricted to 2-regular elements. On 2-regular elements, $\theta_{s}$ and $\theta_{-s}$ coincide and thus belong to the same block. Since in the present situation all characters have height 0 and any block of defect $d$ has at most $2^{l}$ (ordinary) characters [8], $\theta_{s}$ and $\theta_{-s}$ are the ordinary characters of a block of defect 1 . Since there is only one modular character in their block (cf. [10, p. 617]), the Cartan matrix must be [2] and the decomposition matrix

$$
\left[\begin{array}{l}
1 \\
1
\end{array}\right] .
$$

Thus $\theta_{s}$ restricted to 2-regular elements provides a modular character. Finally, since the only 2-regular class of defect 2 is that of 1 , the principal 2-block is the only block of defect 2 . Its ordinary characters are $\theta_{1}, \theta_{2}, \theta_{3}, \theta_{4}$; there are three modular characters not counted so far, and they must be in this block.

4. We consider the principal 2-block more closely. There are two 2-sections (see BII) in $L F(2, q)$, those of 1 and $J^{\prime}$. By BII 7D there are four columns of (nonzero) generalized decomposition numbers for the principal 2-block for these two sections, three for that of 1 and one for $J^{\prime}$. The modular character of the 
centralizer of $J^{\prime}$ in $L F(2, q)$ involved must be the principal (identity) character. That centralizer is $\left\langle J_{0}, S_{0}\right\rangle$ where $J_{0}^{2}=1$ and $J_{0} S_{0} J_{0}=S_{0}^{-1}$. If $S_{0}^{b}$ is 2-regular, $J^{\prime} S_{0}^{b}$ is an odd power of $S_{0}$. The column of decomposition numbers for $\theta_{1}, \theta_{2}, \theta_{3}, \theta_{4}$, and $J^{\prime}$ is then $(1,-e,-e, e)$. The other three columns form a basis for the set of columns with rational integral entries orthogonal to this one (see [4]); by replacing the three modular characters by integral combinations, we may assume these columns are

$\begin{array}{rrr}1 & 0 & 0 \\ 0 & -e & 0 \\ 0 & 0 & -e \\ -e & -e & -e\end{array}$.

The functions so obtained are $\theta_{1},-e \theta_{2},-e \theta_{3}$ restricted to 2-regular elements. They will be denoted by $\phi_{1}^{J}, \phi_{2}^{J}, \phi_{3}^{J}$. $\phi_{2}^{J}$ and $\phi_{3}^{J}$ are algebraically conjugate (complex conjugate if $e=-1$ ). The Cartan matrix is then

$$
\left[\begin{array}{lll}
2 & 1 & 1 \\
1 & 2 & 1 \\
1 & 1 & 2
\end{array}\right] .
$$

5. The characters of the entire centralizer $C(J)$ are obtained from the fact that $C(J)=\langle J\rangle L$, where $L$ is isomorphic to $L F(2, q)$ (and we now use the letters used for $L F(2, q)$ for elements of $L)$. The modular characters of $C(J)$ are those of $L$. To each ordinary character $\chi$ of $L$ correspond two of $C(J)$. One (also labelled $\chi$ ) is $\chi$ on $L$ and has value $\chi(A)$ on $J A, A \in L$. The other, labelled $\chi^{\prime}$, is $\chi$ on $L$ but $-\chi(A)$ on $J A$. $\chi$ and $\chi^{\prime}$ are in the same 2-block, but if $\chi_{1}$ and $\chi_{2}$ (of $L$ ) are not, neither are $\chi_{1}^{\prime}$ and $\chi_{2}^{\prime} . \chi$ and $\chi^{\prime}$ have the same (ordinary) decomposition numbers (see BII).

6. We now plan to use the theory of exceptional characters (see [19] and the expositions in [6] and [10]). If $H$ is a subgroup of $G$, a collection of conjugate classes $K_{1}, \cdots, K_{m}$ of $H$ is a collection of special classes if whenever $X \in K_{i}$ and $A^{-1} X A \in H$ for some $A \in G$, then in fact $A \in H$.

Suppose $\theta_{1}, \cdots, \theta_{n}$ is a family of irreducible characters of $H, n>1$, maximal with the property that $\theta_{1}(X)=\cdots=\theta_{n}(X)$ for all $X$ in $H$ not members of special classes. Then:

a. There exist $n$ irreducible characters $\chi_{1}, \cdots, \chi_{n}$ of $G$ (the exceptional characters) such that $\chi_{i} \mid H=\varepsilon \theta_{i}+\theta$, where $\varepsilon= \pm 1$ (independent of $i$ ) and $\theta$ is a character of $H$ containing the $\theta_{i}$ with equal multiplicity. If $\chi$ is an irreducible character of $G$ not one of the $\chi_{i}, \chi \mid H$ contains the $\theta_{i}$ with equal multiplicity.

b. If $A \in G$ is not conjugate to any member of any $K_{i}$, then $\chi_{1}, \cdots, \chi_{n}$ all have the 
same value on $A$. (In particular, if $G \neq H$, the class of 1 cannot be special and all $\chi_{i}$ have the same degree.)

Moreover, two different maximal families lead to disjoint families of exceptional characters.

Property IV of $G$ makes the classes of the $R^{a} \neq 1$ in $C(J)$ special; the classes of the $J R^{a}$ also become special. The families of the $\theta_{r}$ and the $\theta_{r}^{\prime}$ provide then two families of exceptional characters for $G$, the $\eta_{r}$ and the $\eta_{r}^{\prime}: \eta_{r} \mid C(J)=\varepsilon \theta_{r}+\eta$, $\eta_{r}^{\prime} \mid C(J)=\varepsilon^{\prime} \theta_{r}^{\prime}+\eta^{\prime}, \varepsilon= \pm 1, \varepsilon^{\prime}= \pm 1$, and the multiplicity of $\theta_{r}$ in $\eta$ and $\theta_{r}^{\prime}$ in $\eta^{\prime}$ is independent of $r$. Each family has $(q-2+e) / 4$ members.

7. The characters $\eta$ and $\eta^{\prime}$ actually vanish on the special classes of $C(J)$. For, the orthogonality relations in $L$, applied to $R^{a} \neq 1$ and $T^{\prime}$ give $\sum \theta_{r}\left(R^{a}\right)=e$, summed over the $\theta_{r}$. Then also $\sum \theta_{r}^{\prime}\left(R^{a}\right)=e$. From the constant multiplicity property above and the fact that the other characters of $C(J)$ have rational integral values on $R^{a}$ independent of $a, \chi\left(R^{a}\right)$ is a rational integer independent of $a$ for any nonexceptional irreducible character $\chi$ of $G$. The same is true for $\eta$ and $\eta^{\prime}$.

Condition IV implies that $C\left(R^{a}\right)$, for $R^{a} \neq 1$, is the centralizer in $C(J)$ of $R^{a}$. Thus $c\left(R^{a}\right)=q+e$. Now the order of the centralizer of any element of $G$ is the sum of the squares of the absolute values of the irreducible characters of $G$ on that element. Let $\xi_{1}$ be the identity character of $G$ and let $\xi_{j}$ in general denote a nonexceptional character of $G$. Then this relation for $R^{a}$ becomes

$$
q+e=\sum_{r}\left|\eta_{r}\left(R^{a}\right)\right|^{2}+\sum_{r}\left|\eta_{r}^{\prime}\left(R^{a}\right)\right|^{2}+\sum_{j}\left|\xi_{j}\left(R^{a}\right)\right|^{2} .
$$

Let $\eta\left(R^{a}\right)=x, \eta^{\prime}\left(R^{a}\right)=x^{\prime}$. Then

$$
\begin{aligned}
\sum_{r}\left|\eta_{r}\left(R^{a}\right)\right|^{2} & =\sum_{r}\left|\varepsilon \theta_{r}\left(R^{a}\right)+x\right|^{2} \\
& =\sum_{r}\left|\theta_{r}\left(R^{a}\right)\right|^{2}+\varepsilon x \sum_{r}\left(\theta_{r}\left(R^{a}\right)+\overline{\theta_{r}\left(R^{a}\right)}\right)+(q-2+e) x^{2} / 4 .
\end{aligned}
$$

The first sum may be evaluated by using this same centralizer relation in $L$, yielding $\Sigma_{r}\left|\theta_{r}\left(R^{a}\right)\right|^{2}=\frac{1}{2}(q+e)-2$. The middle term is $2 e \varepsilon x$. Using the same procedure on the $\eta_{r}^{\prime}$ terms, and the fact that $\theta_{r}^{\prime}$ and $\theta_{r}$ agree on $R^{a}$, one finally obtains

(1) $4=\left(\frac{1}{4}(q-2+e) x^{2}+2 e \varepsilon x\right)+\left(\frac{1}{4}(q-2+e) x^{\prime 2}+2 e \varepsilon^{\prime} x^{\prime}\right)+\sum_{j}\left|\xi_{j}\left(R^{a}\right)\right|^{2}$.

Because the terms in the last sum are integers (one of which is 1) and because $q \geqq 27$, it can only be that $x=x^{\prime}=0$. Moreover, four of the $\xi_{j}$ must have values \pm 1 on $R^{a}(\neq 1)$ independent of $a$; call them $\xi_{1}, \xi_{2}, \xi_{3}, \xi_{4}$.

Quite similarly $\eta\left(J R^{a}\right)=\eta^{\prime}\left(J R^{a}\right)=0$, and these same four characters $\xi_{1}, \xi_{2}, \xi_{3}$, and $\xi_{4}$ have values \pm 1 on $J R^{a}(\neq J)$ because $\xi_{j}\left(J R^{a}\right) \equiv \xi_{j}(R)(\bmod 2)$.

Note that $\sum_{r} \eta_{r}\left(R^{a}\right)=\Sigma_{r} \eta_{r}{ }^{\prime}\left(R^{a}\right)=\varepsilon e$.

8. We wish now to show that there are two possibilities for the number of characters for the principal 2-block, namely seven and eight. 
If $B$ is a 2-block of positive defect $d$ of $G$, a defect group may be taken with $J$ in its center. Then (see [1]) there is a block $b$ of defect $d$ of $C(J)$ such that $B=b^{G}$ (cf. BII) and for every ordinary character $\chi$ of $B$ there is a modular character $\phi^{J}$ of $b$ such that the decomposition numbers of $\chi$ for the section of $J$ and $\phi^{J}$ is not 0 .

From BII 6A the decomposition numbers for a character in the principal 2-block $B_{0}$ of $G$ for the section of $J$ can be nonzero only for blocks $b$ of $C(J)$ for which $b^{G}=B_{0}$. Here, as $\langle J\rangle$ is in the defect group of each block of $C(J)$ (BI 9F), the centralizer of such a defect group is in $C(J)$. Then $b^{G}=B_{0}$ iff $b$ is the principal 2-block of $C(J)$ (see [4] or [7]). The Cartan matrix of the principal 2-block of $C(J)$ (relative to $\phi_{1}^{J}, \phi_{2}^{J}, \phi_{3}^{J}$ of paragraph 4 ) is

$$
\left[\begin{array}{ccc}
4 & 2 & 2- \\
2 & 4 & 2 \\
2 & 2 & 4
\end{array}\right],
$$

double that of $L$.

There are thus three columns of decomposition numbers for the principal 2-block $B_{0}$ of $G$ for the section of $J$ and they correspond to $\phi_{1}^{J}, \phi_{2}^{J}, \phi_{3}^{J}$. The inner product of the $i$ th and $j$ th columns is the $i j$ entry in this Cartan matrix. Because $J$ is of order 2, the columns consist of rational integers, and the inner product condition forces the entries to be $0, \pm 1$, or \pm 2 . But no \pm 2 can appear, for it would then be the only entry in its column. However, as these columns are orthogonal to the columns of degrees of the characters in $B_{0}$, a single-entry column is out.

From the first part of the discussion, no row can be entirely 0's. On the basis of the preceding remarks there are two possible patterns:

$\begin{array}{cccccc}\phi_{1}^{J} & \phi_{2}^{J} & \phi_{3}^{J} & \phi_{1}^{J} & \phi_{2}^{J} & \phi_{3}^{J} \\ 1 & 0 & 0 & 1 & 0 & 0 \\ \delta_{2} & 0 & 0 & \delta_{2} & \delta_{2} & 0 \\ \delta_{3} & \delta_{3} & \delta_{3} & \delta_{3} & 0 & \delta_{3} \\ \delta_{4} & \delta_{4} & \delta_{4} & \delta_{4} & \delta_{4} & \delta_{4} \\ 0 & \delta_{5} & 0 & 0 & \delta_{5} & 0 \\ 0 & \delta_{6} & 0 & 0 & 0 & \delta_{6} \\ 0 & 0 & \delta_{7} & 0 & \delta_{7} & \delta_{7} \\ 0 & 0 & \delta_{8} & & & \end{array}$


Each $\delta_{i}$ is \pm 1 . The first four characters in either case are $\xi_{1}, \xi_{2}, \xi_{3}$, and $\xi_{4}$, of paragraph 7. The 2-Sylow subgroup of $G$ is its own centralizer (from the structure of $L$ ) so that by BI $6 \mathrm{D}$ this block $B_{0}$ is the only one of 2-defect 3 .

9. Before analyzing the principal 2-block in detail, we derive information on the other 2-blocks of $G$ of positive defect. First we consider blocks of defect 1 .

Let $\phi_{r}^{J}$ be the modular character of $C(J)$ given by $\theta_{r}$ (paragraph 3). The defect of the block $b_{r}$ of $\phi_{r}^{J}$ is 1 and the Cartan matrix is [2]. As $C(J)$ is the normalizer of $\langle J\rangle$ in $G$, BI 10B says the map $b_{r} \rightarrow b_{r}^{G}$ produces a 1-1 correspondence between the blocks $b_{r}$ of $C(G)$ of defect 1 and those of $G$ having defect group $\langle J\rangle$. As the involutions of $G$ are all conjugate, this means all blocks of $G$ of defect 1 . The column of decomposition numbers for the section of $J$ and $\phi_{r}^{J}$ has then two nonzero entries, \pm 1 . By the orthogonality to the degrees, one is 1 , the other -1 . From BII 6A the corresponding characters are in $b_{r}^{G}$. No other block $b$ of $C(J)$ has $b^{G}=b_{r}^{G}$, for either $b$ is another block of defect 1 or it, and therefore $b^{G}$, has defect greater than 1 . Then all the other columns (for the section of $J$ ) have 0 's for these two characters. From paragraph 8, no character in $b_{r}^{G}$ has a row of 0 's for its decomposition numbers for $J . b_{r}{ }^{G}$ thus contains exactly two characters. From $\eta_{r}(J A)=\varepsilon \phi_{r}^{J}(A)+\eta(J A)$ for $A \in L$, and the equal multiplicity result for $\eta, \eta_{r}$ decomposed for the section of $J$ involves $\phi_{r}^{J}$ with a multiplicity different from that of the other modular characters of defect 1 of $C(J)$. Because there are at least three such characters, $\eta_{r}$ must be in $b_{r}^{G}$. Similarly $\eta_{r}^{\prime}$ is in this block, and $\varepsilon=\varepsilon^{\prime}$ : the column for $\phi_{r}^{J}$ is $(\varepsilon,-\varepsilon)$.

10. Consider now 2-blocks of $G$ of defect 2. Because the involutions of $L$ are all conjugate in $L$, all groups of order 4 of $G$ are conjugate. Any group of order 4 in $L$ is normalized but not centralized by an element of order 3 (cf. [11]). By a conjugation, we may take $\left\langle J, J^{\prime}\right\rangle$ to be the defect group of any 2-block of defect 2, with an involution $J^{\prime \prime}$ such that $\left\langle J, J^{\prime}, J^{\prime \prime}\right\rangle$ is of order 8 . Then $C\left(J, J^{\prime}\right)=\left\langle J, J^{\prime}, J^{\prime \prime}, S\right\rangle$ and $J^{\prime \prime} S J^{\prime \prime}=S^{-1} .\langle S\rangle$ is the commutator subgroup of $C\left(J, J^{\prime}\right)$.

From BI 12A the blocks in $G$ with defect group $\left\langle J, J^{\prime}\right\rangle$ are in 1-1 correspondence with families of associated characters of defect 0 of $C\left(J, J^{\prime}\right) /\left\langle J, J^{\prime}\right\rangle$. (The relatively prime condition of BI 12A is automatic here.) Let $\theta$ be a character of one of these families, belonging to the block $b$ when regarded as a character of $C\left(J, J^{\prime}\right)$. If $\theta$ 's family corresponds to the block $B$ of $G$, then $b^{G}=B$ (BII $\left.2 D\right)$. The 2-blocks of $C(J)$ of defect 2 also have defect group $\left\langle J, J^{\prime}\right\rangle$. By BI 10B there is a 1-1 correspondence between blocks of $C(J)$ with defect group $\left\langle J, J^{\prime}\right\rangle$ and blocks of defect 2 of the normalizer in $C(J)$ of $\left\langle J, J^{\prime}\right\rangle$, i.e., $C\left(J, J^{\prime}\right)$. If the block $b_{1}$ of $C\left(J, J^{\prime}\right)$ corresponds to the block $B_{1}$ of $C(J)$, then $b_{1}^{C(J)}=B_{1}$. From BII $2 \mathrm{G}$ the blocks of $C\left(J, J^{\prime}\right)$ and $C\left(J, J^{\prime}\right) \mid\left\langle J, J^{\prime}\right\rangle$ are in 1-1 correspondence: a character of $C\left(J, J^{\prime}\right)\left\langle\left\langle J, J^{\prime}\right\rangle\right.$ belonging to a given block belongs to the corresponding block when regarded as a character of $C\left(J, J^{\prime}\right)$. As the characters of defect 0 of $C\left(J, J^{\prime}\right) /\left\langle J, J^{\prime}\right\rangle$ are in 1-1 correspondence with blocks of defect 0 , each block 
of defect 2 of $C\left(J, J^{\prime}\right)$ contains exactly one such character (as a character of $\left.C\left(J, J^{\prime}\right)\right)$.

Thus if $b$ is a 2-block of $C\left(J, J^{\prime}\right)$ of defect $2, b^{G}$ and $b^{C(J)}$ are blocks of defect 2; from BII $2 \mathrm{C},\left(b^{C(J)}\right)^{G}=b^{G}$. Now $C\left(J, J^{\prime}\right) /\left\langle J, J^{\prime}\right\rangle$ is isomorphic to $\left\langle J^{\prime \prime}, S\right\rangle$. If $A$ is an element of order 3 normalizing but not centralizing $\left\langle J, J^{\prime}\right\rangle$, condition $\mathrm{V}$ on $G$ yields $A^{-1} S A \neq S^{-1}$. $\left\langle J^{\prime \prime}, S\right\rangle$ is a dihedral group. The two-dimensional irreducible characters are induced from the nontrivial one-dimensional characters of $\langle S\rangle$. If $\lambda$ generates the character group of $\langle S\rangle$ and if $A^{-1} S A=S^{n}$, then $\lambda^{A}$ (the character associated to $\lambda$ by $A$ ) is $\lambda^{n}$. As $\lambda^{m}$ and $\lambda^{-m}$ induce the same character, we may let the class $\left\{S^{m}, S^{-m}\right\}$ of $\left\langle J^{\prime \prime}, S\right\rangle$ correspond to the character induced by $\lambda^{m}$; this correspondence is preserved under the action of $A$. Since $A$ moves at least one class of $\langle S\rangle$, it moves at least one two-dimensional character; that is, there is a character $\theta$ of degree 2 generating a family of three associate characters ( $A$ generates the normalizer of $\left\langle J, J^{\prime}\right\rangle$ over $\left.C\left(J, J^{\prime}\right)\right)$. Thus there is at least one set of three blocks $b_{4}, b_{5}, b_{6}$ of $C(J)$ of defect 2 for which $b_{4}^{G}=b_{5}^{G}=b_{6}^{G}=B_{2}$, a block of $G$ of defect 2. These are the only blocks $b$ of $C(J)$ with $b^{G}=B_{2}$. Let $\phi_{4}^{\mathbf{J}}, \phi_{5}^{\mathbf{J}}, \phi_{6}^{J}$ be the modular characters for $b_{4}, b_{5}, b_{6}$, respectively (cf. paragraph 3). The Cartan matrix for each of these blocks is [4] (the ordinary characters of any one are $\theta_{s}, \theta_{s}^{\prime}, \theta_{-s}, \theta_{-s}^{\prime}$ for suitable $s$ ).

If $\chi$ is an ordinary character of $B_{2}$, the Cartan matrix [4] means no decomposition number for the section of $J$ and $\chi$ is \pm 2 , otherwise that is the only entry in its column (cf. paragraph 8 ). The entries are 0 or \pm 1 , then. Because the defect is $2, B_{2}$ contains at most four ordinary characters [8]. There must be then exactly four characters, and the orthogonality to the column of degrees means that they all have the same degree and the following decomposition $(\delta= \pm 1)$ :

$\begin{array}{lrrr} & \phi_{4}^{J} & \phi_{5}^{J} & \phi_{6}^{J} \\ \xi_{11} & \delta & \delta & \delta \\ \xi_{12} & -\delta & -\delta & -\delta \\ \xi_{13} & -\delta & \delta & -\delta \\ \xi_{14} & \delta & -\delta & -\delta .\end{array}$

11. A formula of Brauer [5] will be applied several times to the principal block and the block of defect 2 above. The formula is given not in its most general form, but in the form it takes in the present case. Let $b$ be a 2-block of $C(J)$ and $\phi_{j}^{J}$ any member of a basis of the modular characters of $b$, i.e., any member of the set of transforms of the modular characters by a unimodular transformation (integral coefficients ). Let $B=b^{G}$. Let $d_{i j}^{J}$ and $d_{i j}^{\prime J}$ be the decomposition numbers for the section of $J$ relative to $\phi_{j}{ }_{j}$ for the characters $\chi_{i} \in B$ and $\chi_{i}^{\prime} \in b$, respectively. Let $h_{i}=\chi_{i}^{\prime}(J) / q\left(q^{2}-1\right)+\chi_{i}^{\prime}\left(J^{\prime}\right) / 2(q-e)+\chi_{i}^{\prime}\left(J J^{\prime}\right) / 2(q-e)$. Then 


$$
g \sum_{\chi_{i} \in B}\left(\chi_{i}(J)\right)^{2} d_{i j}^{J} / \chi_{i}(1)=\left(q\left(q^{2}-1\right)\right)^{3} \sum_{\chi^{\prime} i \in b}\left(h_{i}\right)^{2} d_{i j}^{\prime J} / \chi_{i}{ }^{\prime}(1) .
$$

12. If (2) is applied to the block $B_{2}$ above and the modular character $\phi_{4}^{J}$, there results $\delta g=q^{3}(q-e) \xi_{11}(1)$, forcing $\delta=1$. Generally, if $H$ is a subgroup of $G$ and $\chi$ is a character of $G$, the multiplicity of the principal character of $H$ in $\chi \mid H$ is $|H|^{-1} \Sigma_{A \in H} \chi(A)$. As this must be a rational integer, one obtains $\Sigma_{A \in I} \chi(A) \equiv 0(\bmod |H|)$. For $\xi_{11}$ and $H=\langle R\rangle$ this yields $\xi_{11}(1) \equiv 0$ $\left(\bmod \frac{1}{2}(q+e)\right)$, or, as $\xi_{11}(1)$ is even, $\xi_{11}(1)=(q+e) a, a$ an integer. Thus $g=q^{3}\left(q^{2}-1\right) a$.

13. One further numerical function is useful in discussing the principal block. For $A \in G$, let $\sigma(A)$ be the number of $X \in G$ with $X^{2}=A$.Then $\sigma$ is a class function, so that $\sigma(A)=\sum \sigma_{\chi} \chi(A)$ summed over the characters of $G$. From [12] $\sigma_{\chi}$ is given as follows: if $\chi$ comes from a representation in the real numbers, $\sigma_{\chi}=1$. If $\chi$ is real but the representation cannot be put in the real numbers, $\sigma_{\chi}=-1$. Otherwise, $\sigma_{\chi}=0$. Moreover, if $\chi$ has an odd degree, $\sigma_{\chi} \neq-1$.

If $A \in C(J)$ and $A$ is 2-regular, $\sigma(J A)=0$, or $\Sigma \sigma_{\chi} \chi(J A)=0$. Expressing the $\chi$ 's in terms of the decomposition numbers and modular characters for the section of $J$ and using the independence of the modular characters of $C(J)$, one finds that the column of $\sigma_{x}$ 's is orthogonal to each column of decomposition numbers for the section of $J$. Let $\sigma_{i}=\sigma_{\xi_{i}}$.

14. Certain relations involving the characters of the principal block are valid regardless of which decomposition is correct. For $1 \leqq i \leqq 4$ let $\xi_{i}\left(R^{b}\right)=e_{i}, R^{b} \neq 1$, so that $e_{i}(= \pm 1)$ is independent of $b$ (paragraph 7). The orthogonality relations for the sections of 1 and $J$ imply $1+\delta_{2} e_{2}+\delta_{3} e_{3}+\delta_{4} e_{4}=0$.

The characters of defect 1 all have the same degree $d$, by paragraph 9 and their exceptional nature. The results of paragraph 7 and the orthogonality relations for $R^{b}$ and 1 give $2 \varepsilon e d+1+e_{2} d_{2}+e_{3} d_{3}+e_{4} d_{4}=0$, where $d_{i}=\xi_{i}(1)$. Finally, the orthogonality of degrees and decomposition numbers (in the first column) gives $1+\delta_{2} d_{2}+\delta_{3} d_{3}+\delta_{4} d_{4}=0$.

15. Suppose now there were only seven characters in the principal block. As the algebraic conjugate of any character in this block is also in it [7], $\xi_{2}$ and $\xi_{3}$ are algebraic conjugates (by paragraph 4) and $\delta_{2}=\delta_{3}, d_{2}=d_{3}, e_{2}=e_{3}$, $\sigma_{2}=\sigma_{3}$. Since $\xi_{4}(J)=-\delta_{4} e q, d_{4}$ is odd; as $\xi_{4}$ must be its own complex conjugate (regardless of $e$ ), $\sigma_{4}=1$. By paragraph $13,1+2 \delta_{2} \sigma_{2}+\delta_{4}=0$. If $e=-1, \xi_{2}$ and $\xi_{3}$ are complex conjugate, $\sigma_{2}=0$, and $\delta_{4}=-1$. If $e=1$, each $\xi_{i}$ is real (as the $\phi_{j}^{J}$ are), $\sigma_{2} \neq 0$, and $\delta_{4}=1$. Thus $\delta_{4}=e$.

From the orthogonality of sections using the second column, $\delta_{2} e_{2}+\delta_{4} e_{4}=0$. The relations of paragraph 14 give $\delta_{3} e_{3}=-1$, so $\delta_{4} e_{4}=1$. As $1+2 \delta_{2} d_{2}+\delta_{4} d_{4}=0$ and degrees are positive, $\delta_{2}=-\delta_{4}$. Thus $\delta_{2}=\delta_{3}=-\delta_{4}=-e, e_{2}=e_{3}=e_{4}=e$, and $d_{4}=2 d_{2}-e$. Also, from paragraph 14 , dee $=-2 d_{2}$, forcing $e \varepsilon=-1$.

16. Formula (2) applied to the principal block and $\phi_{1}^{J}$, simplified with the information above (and with $\xi_{2}(J)=\xi_{3}(J)=-\frac{1}{2} \delta_{3} e(q-e)$ ) becomes 


$$
g(d+(q-e))^{2}=q^{2}\left(q^{2}-1\right)^{2}(q+e) d(d-e) .
$$

Using the result of paragraph 12 we obtain

$$
q a(d+(q-e))^{2}=\left(q^{2}-1\right)(q+e) d(d-e) .
$$

Now $q$ is a prime power, so $q \mid d$ or $q \mid(d-e)$. However, if $q \mid(d-e)$, the left side of (4) is divisible by $q^{3}$, so in fact $q^{3} \mid(d-e)$. Since $(g / c(J))\left(\xi_{2}(J) / d_{2}\right)$ is an algebraic integer (cf. BI), $q^{2} a(q-e) / d$ is a rational integer. As $q^{2}$ is prime to $d$, this means $d \mid a(q-e)$. Since $d \geqq q^{3}+e$, we have $a \geqq\left(q^{3}+e\right)(q-e)^{-1}$. But (4) implies $a<q^{-1}\left(q^{2}-1\right)(q+e)$, and the combination of these two inequalities yields the contradiction $q e<-2 q^{2}+1$.

Thus $q \mid d$. Then $d-e$ is prime to $q$ and to $d+q-e$, so that $(d-e) \mid a$, from (4). Now $\xi_{4}\left(R^{b}\right)=e\left(R^{b} \neq 1\right)$; thus (cf. paragraph 12$) d_{4} \equiv e\left(\bmod \frac{1}{2}(q+e)\right)$ and $d \equiv 2 e\left(\bmod \frac{1}{2}(q+e)\right)$. As $d$ is even, then $(q+e) \mid(d-2 e)$. Since $q$ and $q+e$ are relatively prime, we have $d=b q(q+e)-2 q$ for some $b \geqq 1$. Then the conditions $d-e \leqq a<q^{-1}\left(q^{2}-1\right)(q+e)$ force $b=1, a=d-e$. Substitution in (4) leads to $e=1$. Thus, potentially, $g=q^{3}\left(q^{2}-1\right)\left(q^{2}-q-1\right)$, and $d=q(q-1)$.

17. To rule out this case, we use (2) applied to $\phi_{2}^{J}$. The right-hand side in general is $-e q^{2}\left(q^{2}-1\right)(q+e)(3 q+e)$. The orthogonality of sections (with the second column) gives $\delta_{2} d_{2}+\delta_{4} d_{4}+\delta_{5} d_{5}+\delta_{7} d_{7}=0$. Let $f=\delta_{5} d_{5}$. Then in the present case, $\delta_{7} d_{7}=1-\frac{1}{2} q(q-1)-f$. Substitution into (2) of these values and the values found above (along with $\xi_{5}(J)=-\frac{1}{2} \delta_{5} e(q+e), \xi_{7}(J)=-\delta_{7} e(q+e)$ ) yields the quadratic equation for $f$ :

$$
\begin{gathered}
4\left(q^{2}+7 q+1\right) f^{2}-4(q+1)\left(q^{3}-4 q^{2}+5 q+1\right) f \\
+q(q+1)^{2}(q-2)\left(q^{2}-q-1\right)=0 .
\end{gathered}
$$

However, the discriminant of this quadratic is $16(q+1)^{2}(4 q+1)\left(-3 q^{2}+6 q+1\right)$, which is negative, whereas $f$ is real.

18. Thus the correct number of characters in the principal block is eight. From $\xi_{2}(J)=\delta_{2}, \xi_{3}(J)=-e \delta_{3} q, \xi_{4}(J)=-e \delta_{4} q$, (2) for the character $\phi_{1}^{J}$ becomes

$$
g\left(1+\delta_{2} / d_{2}+\delta_{3} q^{2} / d_{3}+\delta_{4} q^{2} / d_{4}\right)=q^{2}\left(q^{2}-1\right)^{2}(q+e) .
$$

Now in general if $A, B, C$ are in $G$, then the number of times $C$ is a product $A^{\prime} B^{\prime}$ with $A^{\prime}$ conjugate to $A, B^{\prime}$ to $B$, is given by

$$
(A, B ; C)=(g / c(A) c(B)) \sum \chi(A) \chi(B) \overline{\chi(C)} / \chi(1)
$$

summed over the characters of $G$ [9]. (This relation is the source of Brauer's formulas.) In the present case, $(J, J ; C)$ is the number of involutions $J^{*}$ with $J^{*} C J^{*}=C^{-1}$. When $C=R^{b} \neq 1$ this number is $q+e$. Since $\sum \eta_{r}\left(R^{b}\right)$ $=\sum \eta_{r}^{\prime}\left(R^{b}\right)=\varepsilon e$ (paragraph 7$)$, summed over each family, and since $\left(\eta_{r}(J)\right)^{2}$ $=\left(\eta_{r}^{\prime}(J)\right)^{2}=(q-e)^{2}$ (paragraph 9$)$, (7) for $\left(J, J ; R^{b}\right)$ becomes 


$$
(q+e)=\left(g / q^{2}\left(q^{2}-1\right)^{2}\right)\left((q-e)^{2} 2 \varepsilon e / d+1+e_{2} d_{2}+e_{3} q^{2} / d_{3}+e_{4} q^{2} / d_{4}\right)
$$

$\left(d=\eta_{r}(1)=\eta_{r}^{\prime}(1)\right.$.) Elimination of $g$ from (7) and (8) yields

(9) $(q-e)^{2} 2 \varepsilon e / d+e_{2} / d_{2}+e_{3} q^{2} / d_{3}+e_{4} q^{2} / d_{4}=\delta_{2} / d_{2}+\delta_{3} q^{2} / d_{3}+\delta_{4} q^{2} / d_{4}$.

Orthogonality of the second column with the $e_{i}$ 's gives $\delta_{3} e_{3}+\delta_{4} e_{4}=0$. As these numbers are \pm 1 's, we may assume $\delta_{3}=e_{3}, \delta_{4}=-e_{4}$. From $1+\delta_{2} e_{2}+\delta_{3} e_{3}+\delta_{4} e_{4}=0$ (paragraph 14), $\delta_{2}=-e_{2}$. Then the relations of paragraph 14 give eed $=-1-\delta_{3} d_{3}$. If now in (9) we substitute these results, we obtain $1+\delta_{3} d_{3}=e(q-e) \delta_{2} d_{2}$, and $\delta_{4} d_{4}=-e q \delta_{2} d_{2}$. Because the degrees are positive, $\delta_{3}=e \delta_{2}$ and $\delta_{4}=-e \delta_{2}$.

19. The order of $G$ may now be determined up to a determination of $e$. First, if $\xi_{3}$ and $\xi_{4}$ were complex conjugate, then $\delta_{3}=\delta_{4}, e_{3}=e_{4}$. But this would violate $\delta_{3} e_{3}+\delta_{4} e_{4}=0$. Thus each character is real, and as the degrees are odd (from the values on $J$ ), $\sigma_{3}=\sigma_{4}=1$. In addition, $\xi_{2}$ is self-conjugate and of odd degree; thus $\sigma_{2}=1$. Then from paragraph $13,1+\delta_{2}+\delta_{3}+\delta_{4}=0$. From paragraph 18, this is $1+\delta_{2}=0$. Hence $\delta_{2}=-1, \delta_{3}=-e, \delta_{4}=e$ (and $\varepsilon=1$ ). With these values and the fact that $d_{4}=q d_{2}$, (6) becomes

$$
g\left(d_{2}-1\right)^{2}=q^{2}(q+e)^{3}(q-e) d_{2} d_{3} .
$$

Because $e_{2}=1\left(=-\delta_{2}\right)$, restriction of $\xi_{2}$ to $\langle R\rangle$ shows that $(q+e) \mid\left(d_{2}-1\right)$ (since $d_{2}$ is odd). As $d_{3}=(q-e) d_{2}+e, d_{3}$ is then prime to both $q+e$ and $q-e$. Writing $g=q^{3}\left(q^{2}-1\right) a$ (paragraph 12), we have $d_{3} \mid q^{3} a$. Thus (10) in the form $\left(q^{3} a / d_{3}\right)\left(d_{2}-1\right)^{2}=q^{2}(q+e)^{2} d_{2}$ yields $\left(d_{2}-1\right) \mid q(q+e)$. Since $(q+e) \mid\left(d_{2}-1\right),\left(d_{2}-1\right)=b(q+e)$ where $b \mid q$.

On the other hand, as $q^{3}|g, q| d_{2}$ or $q \mid d_{3}$. As $d_{2} \equiv b e+1(\bmod q)$ and $d_{3} \equiv-b(\bmod q)$, it must be that either $b=1($ and $e=-1)$ or $b=q$. But from $\xi_{2}$ restricted to a 2-Sylow subgroup we have $d_{2} \equiv \delta_{2} \equiv-1(\bmod 8)$, or $(2+e) b \equiv 3(\bmod 4)$. If then $b=1, e$ would have to be 1 . Thus $b=q$.

In summary, then: $d_{2}=q(q+e)+1, d_{3}=q^{3}, d_{4}=q^{2}(q+e)+q$, and $g=q^{3}\left(q^{2}-1\right)(q(q+e)+1)$.

20. To determine $e$ (and obtain other degrees) we apply (2) to $\phi_{2}^{J}$ (the right side is given in paragraph 17). Let $f_{5}=\delta_{5} d_{5}, f_{6}=\delta_{6} d_{6}$. Then from the orthogonality of the second column with the column of degrees, $\delta_{3} d_{3}+\delta_{4} d_{4}+f_{5}+f_{6}$ $=0$, giving $f_{5}+f_{6}=-q(q+e)$. Substituting all known so far in (2) yields

$$
12 e f_{5}^{2}+12 e q(q+e) f_{5}+q(q+e)^{2}(q(q+e)+1)=0 .
$$

The discriminant of this quadratic is $16\left(q^{2}-1\right)^{2}(-3 e q)$, and as a rational root is required, we must have $e=-1$ and $q$ an odd power of 3 . Set $q=3 m^{2}$, $m$ a power of 3 . Then $g=q^{3}\left(q^{2}-1\right)(q+1-3 m)(q+1+3 m)$.

$\xi_{7}$ may be taken to be the complex conjugate of $\xi_{5}$, and $\xi_{8}$ that of $\xi_{6} . f_{5}$ and 
$f_{6}$ havepposite signs and are the roots of (11); we choose $\delta_{5}=-1, \delta_{6}=1$ (so $\delta_{7}=-1, \delta_{8}=1$ ).

The decompositions and degrees so far are given below:

\begin{tabular}{lrlrc}
\multicolumn{5}{c}{ Principal 2-Block } \\
$\xi_{1}$ & $\phi_{1}^{J}$ & $\phi_{2}^{J}$ & $\phi_{3}^{J}$ & Degree \\
$\xi_{2}$ & -1 & 0 & 0 & 1 \\
$\xi_{3}$ & 1 & 0 & 0 & $q^{2}-q+1$ \\
$\xi_{4}$ & -1 & -1 & -1 & $q^{3}$ \\
$\xi_{5}$ & 0 & -1 & 0 & $\frac{1}{2}(q-1) m(q+1+3 m)$ \\
$\xi_{6}$ & 0 & 1 & 0 & $\frac{1}{2}(q-1) m(q+1-3 m)$ \\
$\xi_{7}$ & 0 & 0 & -1 & $\frac{1}{2}(q-1) m(q+1+3 m)$ \\
$\xi_{8}$ & 0 & 0 & 1 & $\frac{1}{2}(q-1) m(q+1-3 m)$ \\
& \multicolumn{5}{c}{ 2-Block of Defect 1} \\
& $\phi_{r}^{J}$ & Degree & \\
$\eta_{r}$ & 1 & $q^{3}+1$ & \\
$\eta_{r}^{\prime}$ & -1 & $q^{3}+1$
\end{tabular}

$$
\begin{array}{lrrrc}
\multicolumn{5}{c}{\text { 2-Block of Defect } 2\left(B_{2}\right)} \\
\xi_{11} & \phi_{4}^{J} & \phi_{5}^{J} & \phi_{6}^{J} & \text { Degree } \\
\xi_{12} & -1 & 1 & 1 & (q-1)\left(q^{2}-q+1\right) \\
\xi_{13} & -1 & 1 & 1 & (q-1)\left(q^{2}-q+1\right) \\
\xi_{14} & 1 & -1 & -1 & (q-1)\left(q^{2}-q+1\right) \\
& & & -1 & (q-1)\left(q^{2}-q+1\right)
\end{array}
$$

Chapter II. 2-Blocks of Defect 2

In this chapter the characters of defect 2 for the prime 2 are studied. It is shown that all the blocks of defect 2 have a structure like that of the block $B_{2}$ of paragraph I-10. Moreover, the ordinary characters of these blocks make up the members of two more families of exceptional characters associated with the elements of $\langle S\rangle$.

1. The goal of the first few paragraphs is the determination of the centralizers of elements $S^{a} \neq 1$, and this is done by ruling certain primes out of the orders of these centralizers.

Let $A \in G$ be a 2-regular element whose order is divisible by 3 . Then we shall show that $c(A) \mid 2 q^{3}$. First, $\eta_{r}(A)-\eta_{r}^{\prime}(A)=0$ and $1-\xi_{2}(A)+\xi_{3}(A)-\xi_{4}(A)=0$, from orthogonality with decomposition numbers. As $d_{3}=q^{3}, \xi_{3}$ has defect 0 for 3 and $\xi_{3}(A)=0$. In addition, $\eta_{r}(A)$ and $\eta_{r}^{\prime}(A)$ do not depend on $r$. The orthogonality relations for $A$ and $R$ then give $\eta_{r}(A)=\eta_{r}^{\prime}(A)=1$. Since $(g / c(A))\left(\eta_{r}(A) / \eta_{r}(1)\right)$ is an algebraic integer, $c(A) \mid q^{3}(q-1)$. From property IV, $c\left(R^{a}\right)=q-1$ for 
$R^{a} \neq 1$. Thus no element of order dividing $\frac{1}{2}(q-1)$ centralizes $A$, because $\langle R\rangle$ contains a $p$-Sylow subgroup for any prime $p$ dividing $\frac{1}{2}(q-1)$. Hence $c(A) \mid 2 q^{3}$. It follows that no element of odd order dividing $q^{3}+1$ (except 1 ) is centralized by such an $A$.

2. At this point the structure of the 2-blocks of defect 2 may be established. Let $T_{0} \in N\left(\left\langle J, J^{\prime}\right\rangle\right) \cap C\left(J^{\prime \prime}\right)$ of order 3 give $T_{0}^{-1} J T_{0}=J^{\prime}$ (cf. paragraph $\mathrm{I}-10$ ). $T_{0}$ normalizes $\langle S\rangle$, but, by assumption $\mathrm{V}$, does not centralize $S$. In fact, the result above implies $T_{0}$ centralizes no member of $\langle S\rangle$ but 1 . Because $T_{0}$ conjugates no member of $\langle S\rangle$ (but 1) to its inverse, the discussion in paragraph I-10 implies that each character of degree 2 of $\left\langle J^{\prime \prime}, S\right\rangle$ belongs to a family of three associate characters, and each family leads to a 2-block of defect 2 of $G$. Thus all these blocks have the decomposition given for $B_{2}$ at the end of Chapter $I$. Each has four ordinary characters of degree $(q-1)\left(q^{2}-q+1\right)$.

3. Consider now elements whose orders contain primes dividing $q^{2}-q+1$. (Such elements will be discussed in detail in Chapter IV.) The characters of 2-defect 2 of the previous paragraph and those of defect 1 (paragraph I-9) all have orders divisible by $q^{2}-q+1$. Thus they are of defect 0 for any prime dividing $q^{2}-q+1$ and vanish on the elements under consideration. The same holds for $\xi_{2}$ and $\xi_{4}$. The degrees of $\xi_{5}$ and $\xi_{7}$ being divisible by $q+1+3 m$, these characters have defect 0 for primes dividing $q+1+3 m$. Similarly, $\xi_{6}$ and $\xi_{8}$ have defect 0 for primes dividing $q+1-3 m$.

Let $V$ be an element whose order contains a prime dividing $q+1-3 m$, and $W$ one whose order contains a prime dividing $q+1+3 m$. Both elements are 2-regular, and from the orthogonalities to the decomposition numbers in the principal block along with the results on vanishings, we have $\xi_{3}(V)=\xi_{3}(W)=-1$, $\xi_{5}(V)=\xi_{7}(V)=-1$, and $\xi_{6}(W)=\xi_{8}(W)=1$. In the computation of $(J, V ; W)$ and $(J, W ; V)$ (cf. paragraph I-18) only characters of positive 2-defect need be considered, as the others vanish on $J$. Thus one obtains

(1) $(J, V ; W)=\left(q^{2}+1\right)\left(q^{2}-q+1\right) / c(V)$ and $(J, W ; V)=\left(q^{2}+1\right)\left(q^{2}-q+1\right) / c(W)$.

Now $c(V)$ is odd and $\frac{1}{2}\left(q^{2}+1\right)$ is prime to $g$; thus as the left sides are integers, $c(V) \mid\left(q^{2}-q+1\right)$. Similarly, $c(W) \mid\left(q^{2}-q+1\right)$.

4. The centralizer of $S^{a}(\neq 1)$ may now be determined. If $A \in C\left(S^{a}\right)$ has prime order $p$, then $p \neq 3$ by paragraph 1 and $p \nmid\left(q^{2}-q+1\right)$ by paragraph 3 . As in paragraph $1, p \nmid \frac{1}{2}(q-1)$. Thus the prime factors of $c\left(S^{a}\right)$ are among those of $q+1$. Thus $C\left(S^{a}\right)=\left\langle J, J^{\prime}, S\right\rangle$ of order $q+1$.

5. The theory of exceptional characters will now be applied. $\langle S\rangle$ is normal in $N\left(\left\langle J, J^{\prime}\right\rangle\right)=\left\langle J, J^{\prime}, J^{\prime \prime}, S, T_{0}\right\rangle$. If $A^{-1} S^{a} A \in N\left(\left\langle J, J^{\prime}\right\rangle\right)$ for $A \in G, S^{a} \neq 1$, then $A^{-1} S^{a} A=S^{b}$. Since $C\left(S^{a}\right)=C\left(S^{b}\right), A$ normalizes $\left\langle J, J^{\prime}, S\right\rangle$ and thence $\left\langle J, J^{\prime}\right\rangle$, i.e., $A \in N\left(\left\langle J, J^{\prime}\right\rangle\right)$. As $S^{a}$ is a power of $J S^{a}$ ( $S^{a}$ is of odd order), the same holds if $A^{-1} J S^{a} A \in N\left(\left\langle J, J^{\prime}\right\rangle\right)$. The members of $\langle J S\rangle$ other than 1 and $J$ then represent special classes of $N\left(\left\langle J, J^{\prime}\right\rangle\right)$. 
The group $N\left(\left\langle J, J^{\prime}\right\rangle\right)$ is the semi-direct product of the normal subgroup $\left\langle J, J^{\prime}\right\rangle$ and the subgroup $\left\langle J^{\prime \prime}, T_{0}, S\right\rangle$. The characters may be found following Mackey [15]. Table 2 gives the part of the character table needed. Here $\psi_{t}$ is the character of degree 2 of $\left\langle J^{\prime \prime}, S\right\rangle$ whose value on $S^{a}$ is $t^{a}+t^{-a}$, where $t(\neq 1)$ is a $(q+1) / 4$ th root of unity. If $T_{0}^{-1} S T_{0}=S^{n}$, set $t^{\prime}=t^{n}$ and $t^{\prime \prime}=t^{n^{2}} . \zeta_{u}=\zeta_{t}$ whenever $u=t^{ \pm n^{j}}$, and $\zeta_{t}^{\prime}=\zeta_{t-1}^{\prime}$. There are $(q-3) / 24$ of the $\zeta_{t}$ and $(q-3) / 8$ of the $\zeta_{t}^{\prime}$.

TABLE 2

\begin{tabular}{l|rrcc} 
Value on & 1 & $J$ & $S^{a}$ & $J S^{a}$ \\
\hline $\begin{array}{c}\text { Character } \\
\zeta_{t}\end{array}$ & 6 & 6 & $\psi_{t}\left(S^{a}\right)+\psi_{t^{\prime}}\left(S^{a}\right)+\psi_{t^{\prime \prime}}\left(S^{a}\right)$ & $\zeta_{t}\left(S^{a}\right)$ \\
$\begin{array}{c}\zeta_{t}^{\prime} \\
\text { six of }\end{array}$ & 6 & -2 & $\zeta_{t}\left(S^{a}\right)$ & $\psi_{t^{\prime \prime}}\left(S^{a}\right)-\psi_{t}\left(S^{a}\right)-\psi_{t^{\prime}}\left(S^{a}\right)$ \\
degree one & 1 & 1 & 1 & 1 \\
$\begin{array}{c}\text { two of } \\
\text { degree three }\end{array}$ & 3 & -1 & 3 & -1
\end{tabular}

6. The families $\left\{\zeta_{t}\right\}$ and $\left\{\zeta_{t}^{\prime}\right\}$ serve as the source of exceptional characters (paragraph I-6). As the case $q=27$ is different, let $q>27$ for the moment. The two families are maximal for the special classes of paragraph 5. Let $\left\{\eta_{t}\right\}$ and $\left\{\eta_{t}^{\prime}\right\}$ be the resulting families of exceptional characters. Here $\eta_{t} \mid N\left(\left\langle J, J^{\prime}\right\rangle\right)$ $=\varepsilon_{1} \zeta_{t}+\eta_{1}$ and $\eta_{t}^{\prime} \mid N\left(\left\langle J, J^{\prime}\right\rangle\right)=\varepsilon_{1}^{\prime} \zeta_{t}^{\prime}+\eta_{1}^{\prime}$, with $\varepsilon_{1}= \pm 1, \varepsilon_{1}^{\prime}= \pm 1$. The $\zeta_{t}$ appear in $\eta_{1}$ and in $\eta_{1}^{\prime}$ with equal multiplicity, the $\zeta_{t}^{\prime}$ in $\eta_{1}$ and in $\eta_{1}^{\prime}$ with equal multiplicity. The orthogonality relations on $N\left(\left\langle J, J^{\prime}\right\rangle\right)$ yield $\Sigma \zeta_{t}\left(S^{a}\right)=-1$, $\sum \zeta_{t}^{\prime}\left(S^{a}\right)=-3, \sum \zeta_{t}\left(J S^{a}\right)=-1$, and $\sum \zeta_{t}^{\prime}\left(J S^{a}\right)=1$, for $S^{a} \neq 1$; the sums are over the indicated families. As in paragraph $\mathrm{I}-7$, one finds that the following are all rational integers independent of $a\left(S^{a} \neq 1\right): \eta_{1}\left(S^{a}\right), \eta_{1}\left(J S^{a}\right), \eta_{1}^{\prime}\left(S^{a}\right), \eta_{1}^{\prime}\left(J S^{a}\right)$, and $\chi\left(S^{a}\right)$ and $\chi\left(J S^{a}\right)$ for nonexceptional characters $\chi$.

7. These exceptional characters are exactly the characters of 2-defect 2 as will now be shown. The characters $\psi_{t}$ introduced above are 0 on $J^{\prime \prime}$, and $\left\langle J^{\prime \prime}, S\right\rangle$ has besides the 1-character a linear character which is -1 on $J^{\prime \prime}$ and 1 on $\langle S\rangle$. The orthogonality relations in $\left\langle J^{\prime \prime}, S\right\rangle$ give $\sum \psi_{t}\left(S^{a}\right)=-1, S^{a} \neq 1$. These equations used in the matrix of character values of $\left\langle J^{\prime \prime}, S\right\rangle$ show that the $\psi_{t}$ are a basis for functions on the set $K$ of $(q-3) / 8$ classes of $\left\langle J^{\prime \prime}, S\right\rangle$ represented by the $S^{a} \neq 1$. Moreover, if $\phi_{s}^{J}$ is the modular character of $C(J)$ corresponding to $\theta_{s}$ (paragraph I-3), $-\phi_{s}^{J}\left(S^{a}\right)=\psi_{t}\left(S^{a}\right)$ where $t=s^{2}$. If $\chi$ is a character of $G$, consider the function on $K$ given by $\chi\left(J S^{a}\right)$, expressed as a linear combination of the $\psi_{t}$. From paragraph 6 any nonexceptional character gives equal coefficients, while the exceptional ones do not because $(q-3) / 8>3$. But the characters of 2-defect 2 do not give equal coefficients, either. These characters then coincide with the exceptional ones. Since (when $q>27)(q-3) / 8>6$, a comparison of 
coefficients shows that the ordinary characters of a 2-block of defect 2 are $\eta_{t}$, $\eta_{t}^{\prime}, \eta_{t^{\prime}}^{\prime}$, and $\eta_{t^{\prime \prime}}^{\prime}$, and that the modular characters of $C(J)$ involved are $\phi_{s}^{J}, \phi_{s^{\prime}}^{J}$, and $\phi_{s^{\prime \prime}}^{J}$ where $s^{2}=t,\left(s^{\prime}\right)^{2}=t^{\prime},\left(s^{\prime \prime}\right)^{2}=t^{\prime \prime}$. It follows also that $\varepsilon_{1}=\varepsilon_{1}^{\prime}=-1$. Finally, since the $\phi_{s}^{J}$ are real, the decomposition for the section of $J$ of a conjugate character is the same as that of the original. But as the decompositions for the characters of 2-defect 2 specify the character, these characters are all real.

8. The values of the characters of $G$ other than these exceptional characters on $S^{a} \neq 1$ may now be determinant by a technique like that of paragraph $\mathrm{I}-7$.

Restriction of a nonexceptional character $\chi$ to $\langle S\rangle$ gives $\chi(1) \equiv \chi\left(S^{a}\right)$ $(\bmod (q+1) / 4), S^{a} \neq 1$ (recall $\chi\left(S^{a}\right)$ is independent of $\left.a\right)$. Thus modulo $(q+1) / 4$, $\xi_{2}\left(S^{a}\right) \equiv-\xi_{4}\left(S^{a}\right) \equiv 3, \xi_{3}\left(S^{a}\right) \equiv \xi_{6}\left(S^{a}\right) \equiv \xi_{8}\left(S^{a}\right) \equiv-\xi_{5}\left(S^{a}\right) \equiv-\xi_{7}\left(S^{a}\right) \equiv-1$. Now $c(S)=q+1=\Sigma|\chi(S)|^{2}$, summed over the characters of $G$. Let $\eta_{1}^{\prime}\left(S^{a}\right)=x$, $\eta_{1}^{\prime}\left(S^{a}\right)=y$. The contribution to the sum from the principal 2-block is at least 24 , which is what comes if all the congruences are equalities. If we delete all nonexceptional characters but those of the principal 2-block, then

$$
q+1 \geqq 24+\Sigma\left|\eta_{t}(S)\right|^{2}+\Sigma\left|\eta_{t}^{\prime}(S)\right|^{2} .
$$

This simplifies (by use of character relations in $N\left(\left\langle J, J^{\prime}\right\rangle\right)$ ) to

$$
\left((q-3) x^{2} / 24+2 x\right)+\left((q-3) y^{2} / 8+6 y\right) \leqq 0 .
$$

Since still $q>27$, so that in fact $q \geqq 243$, this forces $x=y=0$. Then not only are all the congruences equalities (because (2) is now an equation) but all nonexceptional characters other than those of the principal 2-block vanish on $S$ and therefore on $S^{a} \neq 1$. Further, $\eta_{t}\left(S^{a}\right)=-\zeta_{t}\left(S^{a}\right), \eta_{t}^{\prime}\left(S^{a}\right)=-\zeta_{t}^{\prime}\left(S^{a}\right)$.

9. Suppose now that $q=27$. Then only the $\zeta_{t}^{\prime}$ furnish exceptional characters. All powers of $S$ are conjugate (except 1$)$ and $\zeta_{t}(S)=-1$. The argument of paragraph 7 still implies that the exceptional characters are the last three characters of the single block of 2-defect 2. The first character of that block, now being nonexceptional, has a rational integral value on $S$. The rest then have the same value, from the orthogonality to the decomposition numbers for $J$. The same sort of argument as in paragraph 8 , but without the quadratic discussion, yields again that those congruences are equalities and that the common value on $S$ for the characters of 2-defect 2 is \pm 1 . The congruence obtained from the restriction to $\langle S\rangle$ shows it to be 1 . (The first character will still be labelled $\eta_{t}$.)

\section{Chapter III. The 3-Sylow Subgroup}

In this chapter the results of the main theorem related to the 3-Sylow subgroup are obtained, along with values of characters on the various 3-elements. One of the main tools is the fact that $\langle J R\rangle$ can be included in the normalizer of 3-groups used in a step-by-step build-up of the 3-Sylow subgroup; the rough effect is that $q$ itself behaves like a prime (this kind of behavior is common in families of simple groups). 
1. First, some character values: let $X \neq 1$ be any member of $G$ of order a (positive) power of 3 . Since $X$ is 2-regular, all characters of a 2-block of defect 2 have the same value on $X$; because these characters are exceptional, all characters of 2-defect 2 have the same value on $X$. Let $b(X)$ be this value. From the principal 2-block, $\xi_{2}(X)=1-\xi_{4}(X), \xi_{5}(X)-\xi_{6}(X)=\xi_{7}(X)-\xi_{8}(X)=-\xi_{4}(X)$ (recall $\left.\xi_{3}(X)=0\right)$. These relations and the orthogonality of $X$ and $S$ give $b(X)=2 \xi_{4}(X)-1$.

Let $F$ be the group of all $T(x), x \in F_{q}$ (paragraph I-1) and let $T=T(1)$. Restriction of the real character $\xi_{4}$ to $F$ gives $\xi_{4}(T)=a q$, where $a$ is a rational integer. As $\xi_{4}(J T)=0, a$ is even. From above, $b(T)=2 a q-1$. The contribution to $c(T)=\Sigma|\chi(T)|^{2}$ from characters of 2-defect 2 is then $(q-3)(2 a q-1)^{2} / 6$. As $c(T) \leqq 2 q^{3}$ (paragraph II-1) and $a$ is even, $a$ must be 0 . Then $\xi_{2}(T)=1$, $\xi_{4}(T)=0$, and $b(T)=-1$. Then for $(T, T ; R)$ one obtains the integer $(q-1)\left(q^{2}+1\right) q^{4} / c(T)^{2}$, implying $c(T) \mid 2 q^{2}$. In particular, $T$ cannot be a central element of a 3-Sylow subgroup of $G$.

2. We now start "building'" a 3-Sylow subgroup. Since $C(T) \supseteq C(F), C(F)$ (which contains $J$ ) has order twice a power of 3, and its 3-Sylow subgroup $P_{1}$ is normal. $\langle R\rangle$ normalizes $F$ (see [11]) and therefore $C(F)$. Consequently $\langle R\rangle$ normalizes $P_{1}$. In general, if $\langle R\rangle$ normalizes the 3 -group $H$, no nonidentity element of $\langle R\rangle$ centralizes a nonidentity element of $H$. If $|H|=h$, then $(q-1) / 2$ divides $h-1$, or, as $h$ is a power of 3 and odd, $h \equiv 1(\bmod (q-1))$. Since $h \mid q^{3}$, $h$ can only be $1, q, q^{2}$, or $q^{3}$.

If $X$ is in the center of some 3-Sylow subgroup of $G$ containing $F$, then $X$ is also in $P_{1}$. Moreover, if $X \neq 1$, then $X \notin F$, for the nonidentity elements of $F$ are conjugate to $T$ or $T^{-1}\left(-1\right.$ is not a square in $\left.F_{q}\right)$. Thus there are central elements of order 3 of $P_{1}$ not in $F$. Thus $P_{1} \neq F$ and so $\left|P_{1}\right|=q^{2}$. As $\langle R\rangle$ normalizes the center of $P_{1}, P_{1}$ must be Abelian. Since $F$ is elementary Abelian, so is $P_{1}$. Finally, $c(T)$ must now be $2 q^{2}$.

3. Certain character values may now be made more precise. Let $X \neq 1$ be an element in the center of a 3-Sylow subgroup of $G$. Because the central elements of any 3-Sylow subgroup of $G$ containing $F$ are in $P_{1}$, it follows that $X$ is of order 3 and $c(X)=q^{3}$. (X cannot centralize an involution.) Then one finds

$$
(X, T ; R)=(q-1)\left(q^{3}+q-(q+1) \xi_{4}(X)\right) / 2 q^{2} .
$$

As this is an integer, $\xi_{4}(X)$ is a rational integer and $\xi_{4}(X)=q+a q^{2}$ for some rational integer $a$. Since $\left|\xi_{4}(X)\right|^{2}<q^{3}, a=0$. Thus $\xi_{4}(X)=q, \xi_{2}(X)=1-q$, and $b(X)=2 q-1$ (paragraph 1$)$.

More generally, let $Y$ be any element of order 3 for which $q^{2} \mid c(Y)$ (e.g., $\left.Y \in P_{1}\right)$. Then one finds

$$
(Y, X ; R)=(q-1)\left(q^{3}-q^{2}+q(q+1) \xi_{4}(Y)\right) / c(Y) .
$$

If the power of 3 in $c(Y)$ is $k q^{2}, k \mid q$, it must be that $\xi_{4}(Y)=q(1+a k), a$ a 
rational integer. As $k \geqq 1$ and $(Y, X ; R) \geqq 0, a \geqq-1$. But if $a=-1, k$ must be 1 and $\xi_{4}(Y)=0$. On the other hand, since $\left|\xi_{4}(Y)\right|^{2}<c(Y) \leqq 2 k q^{2}, a \leqq 0$. If $a=0$, then $Y$ is not conjugate to $T$ or $T^{-1}$. The contribution to $c(Y)=k q^{2}$ from the characters of 2-defect 2 then being $(q-3)(2 q-1)^{2} / 6$, which is more than $q^{3} / 3, k$ must be $q$ and $Y$ must be a central element.

In short, either $\xi_{4}(Y)=0$ and $c(Y) \leqq 2 q^{2}$ or $\xi_{4}(Y)=q$ and $c(Y)=q^{3}$.

4. With this information we can establish the center of a 3-Sylow subgroup. In $P_{1}$, consider the set of elements $X \neq 1$ such that $X$ is in the center of some 3-Sylow subgroup of $G$ containing $F$. Although these elements and 1 do not form a subgroup a priori, nevertheless they are permuted under conjugation by the group $\langle J R\rangle$ and no such element is fixed by a member of $\langle J R\rangle-\langle 1\rangle$. There are then $a(q-1)$ such elements, $a>0$ an integer. The multiplicity of the identity in $\xi_{2} \mid P_{1}$ is then, from paragraph $3,\left[\left(q^{2}-q+1\right)+a(q-1)(1-q)\right.$ $\left.+\left(\left(q^{2}-1\right)-a(q-1)\right)\right] / q^{2}=2-a+(a-1) / q$. As this must be a non-negative integer, $a$ can only be 1 .

If $X$ is one of these $q-1$ central elements, so is $X^{-1}$, and for some $A \in\langle J R\rangle$, $A^{-1} X A=X^{-1}$. Since $A^{2}$ then centralizes $X, A=J$. In general, if $\langle J\rangle$ normalizes the odd subgroup $H$ and $H^{\prime}$ is the set of elements conjugated to their inverses by $J$, then $|C(J) \cap H|\left|H^{\prime}\right|=|H|$. With $H=P_{1}$ there are then $q-1$ nonidentity elements of $P_{1}$ inverted by $J$. Thus the $q-1$ central elements are exactly these elements. Since they all commute, they and 1 form a group $C$ of order $q$, which will turn out to be the center of a 3-Sylow subgroup.

The set $C-\langle 1\rangle$ can be characterized as the set of $X \in P_{1}, X \neq 1$, such that $X$ is in the center of some 3 -Sylow subgroup of $G$ containing $P_{1}$, for that set is contained in $C-\langle 1\rangle$ and is also permuted by conjugation by $\langle J R\rangle$. Consequently, anything normalizing $P_{1}$ normalizes $C . P_{1}$ being properly normal in some 3-group of $G, P_{1}$ is not a 3-Sylow subgroup of $N\left(P_{1}\right)$. An odd prime factor $p$ of $q^{3}+1$ cannot divide $N\left(P_{1}\right)$, for an element of order $p$ would normalize $C$ and thence, as $p \nmid(q-1)$, centralize a member of $C-\langle 1\rangle$. Quite similarly, there is no 4-group in $N\left(P_{1}\right)$. Thus $\left|N\left(P_{1}\right)\right|=k q^{2}(q-1)$ where $k \mid q, k>1$. Now for any prime $p$ dividing $q-1$, the $p$-Sylow subgroup of $N\left(P_{1}\right)$ is in the center of its normalizer in $N\left(P_{1}\right)$ : for $p=2$, this Sylow subgroup is $\langle J\rangle$, and for other $p$ it is $\left\langle R^{a}\right\rangle$ for some $a$, with normalizer $\langle J R\rangle$ (no involution conjugates $R^{a}$ to $R^{-a}$ without centralizing $J$ ). From Burnside's Theorem [13, p. 203], each such Sylow subgroup has a normal complement. The intersection of these complements is a 3-Sylow subgroup $P$ of $N\left(P_{1}\right)$. Since $\langle R\rangle$ then normalizes $P, k=q$ and $P$ is a 3-Sylow subgroup of $G$.

Any central element of $P$ lies in $C$, as $P_{1} \subset P$. But $\langle R\rangle$ normalizes the center of $P$; consequently $C$ must be that center. $P_{1}=C F$ (for $C \cap F=\langle 1\rangle$ ). Just as for $P_{1}$, since anything normalizing $P$ normalizes $C,|N(P)|=q^{3}(q-1)$ and $N(P)=N\left(P_{1}\right)$. Then $P_{1}$ is normal in $N(P)$.

5. Consider the permutation representation of $G$ given by the action of right 
multiplication of cosets of $N(P)$. The degree of the character $\theta$ of this representation is $q^{3}+1$ and it contains $\xi_{1}$ with multiplicity 1 (cf.[13]). None of the $\eta_{r}$ or $\eta_{r}^{\prime}$ can be in $\theta$ because their degrees are too large. If $J_{1}$ is an involution with $J_{1} R J_{1}=R^{-1}$, then $R$ and $J R$ fix $N(P) J_{1} \neq N(P)$, in addition to $N(P)$. If then $\xi_{i}$ has multiplicity $m_{i}$ in $\theta, 2 \leqq 1+m_{2}+m_{3}+m_{4}$ and $2 \leqq 1-m_{2}+m_{3}-m_{4}$. Thus $1 \leqq m_{3}$; as $\xi_{3}$ has degree $q^{3}, \theta=\xi_{1}+\xi_{3}$. Thus [13] this action is doubly transitive. In particular, if $A \in P-\langle 1\rangle, \theta(A)=1$ and $P$ is the only 3-Sylow subgroup containing $A$. There are thus $q^{6}-1$ elements with order a positive power of 3. Finally, if $A \in P-\langle 1\rangle$ and $B^{-1} A B \in P$, then $B \in N(P)$.

6. The group $\langle R\rangle$ normalizes $P$ and no member of $\langle R\rangle-1$ centralizes any member of $P-\langle 1\rangle$. If $H$ is any normal subgroup of $P$ normalized by $\langle R\rangle$, the same conclusion holds for the action of $\langle R\rangle$ on $P / H$. Moreover, if $H$ is a subgroup of $P$ of index $q$ that is normalized by $\langle R\rangle$, the normalizer of $H$ in $P$ is also normalized by $\langle R\rangle$ and must be $P$ itself. Then $P / H$ must be elementary Abelian. Thus $P / C F$ is elementary Abelian. Furthermore, $C F / C$ is in the center of $P / C$. For if not, $C F / C$ meets that center only in 1 ; but then $P / C$ would be Abelian because $C F / C$ is, and $C F / C$ would be in the center after all.

7. We may obtain further character values for central elements of $P$. Because $c(T)=2 q^{2}, T$ has $q(q-1) / 2$ conjugates in $N(P)$, all of which lie in the normal subgroup $P_{1}$ (cf. the last remark of paragraph 5). $T$ and $T^{-1}$ are not conjugate, for if so, $\xi_{5}(T)=\xi_{5}\left(T^{-1}\right)$ would imply that $\xi_{5}(J T)-\xi_{5}\left(J T^{-1}\right)$ belongs to a prime ideal divisor of 2 in the field of $g$ th roots of unity, which is not so. $T^{-1}$ also has $q(q-1) / 2$ conjugates in $P_{1}$, and $P_{1}-C$ thus consists entirely of conjugates of $T$ and $T^{-1}$.

If then $\chi$ is a character of $G$, because the members of $C-\langle 1\rangle$ are all conjugate (paragraph 4), $\chi \mid P_{1}$ gives

$$
\chi(1)+(q-1) \chi(X)+q(q-1)\left(\chi(T)+\chi\left(T^{-1}\right)\right) / 2 \equiv 0\left(\bmod q^{2}\right)
$$

where $X$ stands for a member of $C-\langle 1\rangle$. Since from $\chi \mid F$ one has

$$
(q-1)\left(\chi(T)+\chi\left(T^{-1}\right)\right) / 2 \equiv-1(\bmod q),
$$

one obtains

$$
\chi(X) \equiv \chi(1)\left(\bmod q^{2}\right)
$$

Because $|\chi(X)|^{2} \leqq c(X)=q^{3}$, this congruence can usually be replaced by an equality. Thus $\xi_{5}(X)=\xi_{7}(X)=-(q+m) / 2$ and $\xi_{6}(X)=\xi_{8}(X)=(q-m) / 2$.

8. The rest of this chapter concerns the elements of $P$ outside $P_{1}=C F$. If $Y$ is such an element, $Y^{3} \in P_{1}$ (paragraph 6). However, if $Y^{3}=A B, A \in C$, with $B \in F$ and $B \neq 1$, then $Y$ centralizes $B$, an impossibility. Thus $Y^{3} \in C$. Moreover, if $B \in F$, then $B^{-1} Y B \in Y C$ (paragraph 6). Two different $B$ 's give two different members of $Y C$, for otherwise again $Y$ centralizes a member of $F-\langle 1\rangle$. Thus 
$Y C$ consists of conjugates of $Y .\langle Y\rangle$ is of order 3 or 9 ; in either case $\xi_{2} \mid\langle Y\rangle$ shows $\xi_{2}(Y)$ to be a rational integer congruent to $1(\bmod 3)\left(\xi_{2}\right.$ is real).

That this congruence is an equality comes as follows: there is enough information on orders of centralizers to compute the contribution to $\Sigma\left|\xi_{2}(A)\right|^{2}$, summed over $A \in G$, from elements not conjugate to members of $P-P_{1}$. (The section of $J$ has $3 g / 8$ members and $\xi_{2}$ is 0 on any element whose order is not prime to $q^{2}-q+1$.) The contribution to the sum from elements conjugate to members of $P-P_{1}$ is then $g$ less the first contribution; explicitly, it is $q^{2}(q-1)\left(q^{3}+1\right)$. But this is exactly the number of such elements, and the congruence implies $\xi_{2}(Y)=1$ for all such $Y$.

9. Consider now those $Y \in P-P_{1}$ for which $J Y J=Y^{-1}$. Since $|C(J) \cap P|=q$, there are $q^{2}-1$ members $A$ of $P-\langle 1\rangle$ with $J A J=A^{-1}$ (cf. paragraph 4). Because only $q-1$ of them are in $P_{1}$ (viz., the members of $C-\langle 1\rangle$ ), there are $q^{2}-q$ such $Y$. From $\xi_{2}(Y)=1$ one obtains $\xi_{4}(Y)=0$, and $\xi_{5}, \xi_{6}, \xi_{7}$, and $\xi_{8}$ all have the same value on $Y$. In the computation of $(Y, X ; J)$ only the characters of the principal 2-block enter, for in 2-blocks of defect 1 or 2 all characters have the same value on $Y$ and on $X$ and the sum of the values on $J$ is 0 . Using the values known, one has

$$
(Y, X ; J)=q\left(q^{2}-1\right)\left(q+6 m \xi_{5}(Y)\right) / c(Y) .
$$

Then first of all $\xi_{5}(Y)$ is a rational integer. The centralizer of $J$ permutes (by conjugation) the pairs $\left(Y_{1}, X_{1}\right)$ for which $Y_{1} X_{1}=J, Y_{1}$ conjugate $Y, X_{1}$ to $X$; only the identity can fix a pair. Consequently, $c(Y) \mid\left(q+6 m \xi_{5}(Y)\right)$. If $c(Y)=k q, k$ a power of 3, then $k \geqq 3$, as $\langle Y\rangle C \subseteq C(Y)$. Then $\xi_{5}(Y)=a m, a$ an integer, and $1+2 a=k b, b$ an integer. From $k q=c(Y)>4\left|\xi_{5}(Y)\right|^{2}$ and the fact that $b$ must be odd, one finds $k=3$ and $\xi_{5}(Y)=m$. Thus $C(Y)=\langle Y\rangle C$.

10. The conjugates by members of $P$ of an element $Y$ of paragraph 9 may be determined as follows: as $P / P_{1}$ is Abelian (paragraph 6) the conjugates of $Y$ lie in $Y P_{1}=Y C F$. If $Q_{i}^{-1} Y Q_{i}=Y X_{i} T_{i}, i=1,2$, are two such conjugates, where $Q_{i} \in P, \quad X_{i} \in C$, and $T_{i} \in F$, then if $Q_{2}^{-1} T_{1} Q_{2}=X_{3} T_{1}, \quad X_{3} \in C$, we have $\left(Q_{1} Q_{2}\right)^{-1} Y\left(Q_{1} Q_{2}\right)=Y\left(X_{1} T_{1}\right)\left(X_{2} T_{2}\right) X_{3}$. But for some $T_{3} \in F, T_{3}^{-1} Y T_{3}=Y X_{3}^{-1}$ (paragraph 8), so that $Y\left(X_{1} T_{1}\right)\left(X_{2} T_{2}\right)$ is a conjugate of $Y$. Thus the elements $A$ for which $Y A$ is a conjugate of $Y$ by members of $P$ form a subgroup of $C F$ containing $C$ and therefore of the form $C F(Y), F(Y)$ a subgroup of $F$ of index 3 (since $Y$ has $q^{2} / 3$ conjugates by members of $P$ ). Replacing $Y$ by a conjugate by an element of $\langle R\rangle$, if necessary, we may assume $T \notin F(Y)$.

11. The other elements of $P-P_{1}$ may now be described. Let $Q \in P$ and $Q^{-1} Y Q=Y A, A \in C F(Y)$. If $Q^{-1} T Q=T X_{1}, X_{1} \in C$, and if $T_{1} \in F$ gives $T_{1}^{-1} Y T_{1}=Y X_{1}^{-1}$, then $\left(Q T_{1}\right)^{-1}(Y T)\left(Q T_{1}\right)=Y T A$. Thus $(Y T) C F(Y)$ consists of conjugates of $Y T$ (by members of $P$ ). Since $c(Y T) \geqq 3 q$, this set is all the conjugates of $Y T$ by members of $P$ and $c(Y T)=3 q$. Similarly $c\left(Y T^{-1}\right)=3 q$ and $\left(Y T^{-1}\right) C F(Y)$ are all conjugates of $Y T^{-1}$ by members of $P$. Note that 


$$
Y T^{-1}=\left(J T^{-1}\right)^{-1}(Y T)^{-1}\left(J T^{-1}\right) .
$$

The conjugates of $Y$ by $\langle J R\rangle$ are coset representatives of the cosets of $P_{1}$ in $P$ other than $P_{1}$ (cf. paragraph 6). If $Y_{1} \in P-P_{1}$ some conjugate of $Y_{1}$ by a member of $\langle J R\rangle$ is in $Y P_{1}$. As $Y P_{1}=Y C F(Y) \cup(Y T) C F(Y) \cup\left(Y T^{-1}\right) C F(Y), Y_{1}$ is conjugate to $Y, Y T$, or $Y T^{-1}$. The same sort of discussion shows $Y, Y T$, and $Y T^{-1}$ to be mutually nonconjugate, as an element effecting the conjugation would be in $P$.

$Y$ has order 3 or 9 , from paragraph 8 . However, the order must be 9 : for if $Q \in P_{1}, Q^{-1} Y Q=Y X_{1}$ with $X_{1} \in C$, so that $Q Y Q^{-1}=Y X_{1}^{-1}$. Then $(Y Q)^{3}=Y^{3}$. Thus if $Y^{3}=1, P$ would consist of elements of order 3 or 1 . But in such a group any element commutes with all its conjugates; yet $Y$ has more than $c(Y)=3 q$ conjugates (by members of $P$ ). Thus $Y$ has order 9; the same is true for $Y T$ and $Y T^{-1}$.

Note that the members of $C F(Y)$ are commutator elements $Y^{-1} Q^{-1} Y Q$, $Q \in P$. Thus the derived group, $P^{\prime}$, contains $C$ properly. As $P^{\prime}$ is characteristic, $\left|P^{\prime}\right|=q^{2}$, and, in fact, $P^{\prime}=C F$. Since $P / C F$ is actually elementary Abelian, $C F$ is also the Frattini subgroup of $P$.

12. In this paragraph we show that the roles of $Y T$ and $Y T^{-1}$ may be exchanged. Explicitly, there is an element $R^{a}$ such that if $Y_{1}=R^{-a} Y R^{a}$, then $T_{e} \notin\left(Y_{1}\right)$, just as for $Y$, and $Y_{1} T$ is conjugate in $G$ to $Y T^{-1}$.

For a given $R^{a}, F\left(Y_{1}\right)=R^{-a} F(Y) R^{a}\left(Y_{1}=R^{-a} Y R^{a}\right) . Y_{1} T$ is conjugate to $Y R^{a} T R^{-a}$ and this is to be conjugate to $Y T^{-1}$. Thus $T R^{a} T R^{-a}$ must be in $F(Y)$. If this is so, $T \notin F\left(Y_{1}\right)$, for otherwise $R^{a} T R^{-a} \in F(Y)$, forcing $T \in F(Y)$.

The conjugates of $T$ by the members of $\langle R\rangle$ are the $T(x)$ for which $x \in F_{q}$ is a (nonzero) square. If $R^{a} T R^{-a}=T(x), T R^{a} T R^{-a}=T(1+x)$. Let

$$
H=\{x \mid T(x) \in F(Y)\},
$$

a subgroup of index 3 of the additive group of $F_{q}$. Needed is a nonzero square $x$ with $1+x \in H$. Suppose no such $x$ exists.

0,1 , and -1 represent the cosets of $H$ in $F_{q}$. Then all squares of $F_{q}$ are in $H$ and $1+H$. As -1 is not a square, there are $(q-3) / 4$ squares $b^{2} \neq 0$ with $1+b^{2}$ a square $\left[11\right.$, p. 48]. If $b^{2} \in 1+H, 1+b^{2}$ is a square in $-1+H$. So $b^{2} \in H$. Since there are $(q-1) / 2$ nonzero squares in $F_{q}$, this means the squares (including 0 ) are evenly distributed between $H$ and $1+H$ (and each square in $1+H$ is 1 plus one in $H$ ). Now $-1=a^{2}+b^{2}$ has $q+1$ pairs of solutions in $F_{q}[11$, p. 46]. As $(q+1) / 4>1$, there is a pair with $a^{2} \neq 1, b^{2} \neq 1$. If $a^{2}$ or $b^{2}$ is in $H$, the other is in $-1+H$. So both are in $1+H$. Then there are $c^{2}, d^{2}$ in $H$ with $a^{2}=1+c^{2}$, $b^{2}=1+d^{2}$. Then $c^{2}+d^{2}=0$. As -1 is not a square, $c=d=0$, violating $a^{2} \neq 1, b^{2} \neq 1$. Thus $x$ exists and with it $Y_{1}$.

Chapter IV. Characters of 2-Defect 0

In this chapter the rest of the characters of $G$ are obtained. All but two of them 
are exceptional characters associated with elements in $G$ whose orders contain primes dividing $q^{2}-q+1$.

1. First of all, consider elements of $G$ whose order is not prime to $q^{2}-q+1$. From paragraph II -4 the order of the centralizer of such an element divides $q^{2}-q+1$. Let $V$ be any element whose order is not prime to $q+1-3 m$. Using the values of the characters of the principal 2-block on $V$ determined in paragraph II-4, one finds $(J, J ; V)=q+1-3 m$. The $q+1-3 m$ involutions $J_{1}$ with $J_{1} V J_{1}=V^{-1}$ all lie in the same coset of $C(V)$, so that $c(V) \geqq q+1-3 m$. But $(g / c(V))\left(\xi_{5}(V) / \xi_{5}(1)\right)$ is an algebraic integer; i.e., $c(V) \mid-6 m q^{2}(q+1)(q+1-3 m)$. Consequently $c(V)=q+1-3 m$. $C(V)$ thus has a whole coset of involutions and is therefore Abelian. Quite similarly, if $W$ is an element whose order is not prime to $q+1+3 m, C(W)$ is Abelian of order $q+1+3 m$ and has a coset of involutions.

Thus we obtain two Abelian subgroups $\mathrm{M}^{-}$and $\mathrm{M}^{+}$of orders $q+1-3 m$ and $q+1+3 m$, respectively. Each is the centralizer of any nonidentity member. As each is in addition the centralizer of a Sylow subgroup of $G$ (namely, any of its own Sylow subgroups), any Abelian subgroup of $G$ of order $q+1-3 m$ is conjugate to $M^{-}$, any of order $q+1+3 m$ to $M^{+}$. Any conjugate of $M^{-}$is either $M^{-}$or meets $M^{-}$only in $\langle 1\rangle$, for otherwise a common element has too large a centralizer. Similarly for $M^{+}$.

2. We next determine the orders of the normalizers of $M^{-}$and $M^{+}$. As in paragraph III-8, the number of members of $G$ with orders prime to $q^{2}-q+1$ can be counted: it is $q^{3}\left(q^{2}-1\right)\left(2 q^{2}-q+3\right) / 3$. From paragraph 1 , the normalizers $N\left(M^{-}\right)$and $N\left(M^{+}\right)$are normalizers of Sylow subgroups. Thus $\left[G: N\left(M^{-}\right)\right] \equiv 1$ $(\bmod p)$ for any prime $p$ dividing $q+1-3 m$. If $\left|N\left(M^{-}\right)\right|=(q+1-3 m) a$, then as $p \mid q^{2}-q+1$ and $a$ is prime to $q+1-3 m$, the congruence becomes $a \equiv 6(\bmod$ $p$ ). Since $p \mid\left(q^{3}+1\right)$ and $q^{3}$ is an odd power of $3,-3$ is a square $\bmod p$. Then $p \geqq 7$ and $a \geqq 6$. Similarly, if $\left|N\left(M^{+}\right)\right|=(q+1+3 m) b, b \geqq 6$. Thus the number of elements in all conjugates of $M^{-}$and $M^{+}$(other than 1) is the left side of

$$
(q-3 m) g / a(q+1-3 m)+(q+3 m) g / b(q+1+3 m)=q^{4}\left(q^{2}-1\right)(q-2) / 3
$$

With the inequalities on a and $b$, this forces $a=b=6$.

3. An element of order 3 in $N\left(M^{-}\right)$permutes (by conjugation) the $q+1-3 m$ involutions of paragraph 1 and so centralizes one of them. Taking suitable conjugates we assume $\langle J, T\rangle \subset N\left(M^{-}\right)$. Similarly we assume $\langle J, T\rangle \subset N\left(M^{+}\right)$. The results of paragraph 1 then say that $N\left(\mathrm{M}^{-}\right)$and $N\left(\mathrm{M}^{+}\right)$are Frobenius groups with Frobenius kernels $M^{-}$and $M^{+}$, respectively.

4. Preparatory to applying the exceptional theory we discuss the characters of $N\left(M^{-}\right)$and $N\left(M^{+}\right)$. These groups are semi-direct products of $\langle J, T\rangle$ with $M^{\infty}$ and $M^{+}$, respectively, and this gives their characters (following [15]). Each group has six one-dimensional characters. If $\lambda$ is a (linear) character of $M^{-}, \lambda \neq 1$, 
then the associate character $\lambda^{B}, B \in\langle J, T\rangle-\langle 1\rangle$ is not $\lambda$. For if $\lambda^{B}=\lambda, B$ normalizes the kernel $K$ of $\lambda$ and induces an action on $M^{-} / K$ with no fixed element but 1 . But as the map on $M^{-} / K$ by $\lambda$ is $1-1$, this cannot be. A similar situation holds for $M^{+}$.

Thus for $N\left(M^{-}\right)$there are $(q-3 m) / 6$ 6-dimensional irreducible characters $\theta_{i}^{-}$ which are 0 outside $M^{-}$, and for $N\left(M^{+}\right),(q+3 m) / 6$ 6-dimensional characters $\theta_{i}^{+}, 0$ outside $M^{+}$. The members of $M^{-}-\langle 1\rangle$ form special classes of $N\left(M^{-}\right)$, from paragraph 1 and the fact that $M^{-}$is actually a characteristic subgroup of $N\left(M^{-}\right)$. The characters $\theta_{i}^{-}$form a maximal family for these classes. Similarly the members of $M^{+}-\langle 1\rangle$ form special classes of $N\left(M^{+}\right)$and the $\theta_{i}^{+}$are a maximal family.

5. From the exceptional theory there are then two families (although they must be shown to be distinct, below) of irreducible characters of $G,\left\{\eta_{i}^{-}\right\}$and $\left\{\eta_{i}^{+}\right\}$, for which $\eta_{i}^{-} \mid N\left(M^{-}\right)=\varepsilon^{-} \theta_{i}^{-}+\eta^{-}$and $\eta_{i}^{+} \mid N\left(M^{+}\right)=\varepsilon^{+} \theta_{i}^{+}+\eta^{+}, \varepsilon^{-}= \pm 1$, $\varepsilon^{+}= \pm 1, \eta^{-}$containing the $\theta_{i}^{-}$with equal multiplicity, $\eta^{+}$the $\theta_{i}^{+}$with equal multiplicity. The orthogonality relations in $N\left(M^{-}\right)$give $\sum \theta_{\imath}^{-}(A)=-1$ (summed over $i), A \in M^{-}-\langle 1\rangle$. Similarly $\sum \theta_{i}^{+}(A)=-1, A \in M^{+}-\langle 1\rangle$. Thus if $\chi$ is not one of the $\eta_{i}^{-}$, as $\chi \mid N\left(M^{-}\right)$contains the $\theta_{i}^{-}$with equal multiplicity and the 1-dimensional characters of $N\left(M^{-}\right)$are 1 on $M^{-}, \chi(A)$ is a rational integer independent of $A$. Similarly, if $\chi$ is not an $\eta_{i}^{+}, \chi(A)$ is a rational integer independent of $A \in M^{+}-\langle 1\rangle$.

6. It must be shown that these families are distinct and that the characters in them are new (that is, of 2-defect 0 ).

In general, let $\chi_{1}, \cdots, \chi_{n}$ be a family of exceptional characters of $G$ (not containing the principal character) and $K$ the set of members of $G$ conjugate to members of the special classes for this family (paragraph I-6). Suppose the character $\chi$ of $G$ (irreducible or not) is constant on $K$. Then if the multiplicity of $\chi_{i}$ in $\chi$ is $x_{i}, x_{i}$ does not depend on $i$. For, $g x_{i}=\sum_{A \in K} \chi(A) \overline{\chi_{i}(A)}+\sum_{A \in G-K} \chi(A) \overline{\chi_{i}(A)}$. When $A \in G-K, \chi_{i}(A)$ does not depend on $i$, while in the first sum $\chi(A)$ is constant and $\sum_{A \subseteq K} \chi_{i}(A)=-\Sigma_{A \in G-K} \chi_{i}(A)$ (from the orthogonality to the principal character) does not depend on $i$ either. In particular, if $\chi$ is irreducible, $\chi$ is none of the $\chi_{i}(n>1)$.

Thus, if an irreducible character of $G$ has the same value on all conjugates to members of $M^{-}-\langle 1\rangle$, that character is not one of the $\eta_{i}^{-}$; the same type of conclusion holds for $\mathrm{M}^{+}$. As the members of 2-blocks of positive defect all have this property (for both groups-cf. paragraph II-3), these exceptional characters are indeed new.

If $A \in M^{-}-\langle 1\rangle, A$ is not conjugate to a member of $M^{+}$, and the $\eta_{i}^{+}$all have the same value on $A$. No $\eta_{j}^{-}$is constant on $M^{-}-\langle 1\rangle$, for if so it is not exceptional. If $\eta_{k}^{+}=\eta_{j}^{-}$, then $\eta_{k}^{+}$is not constant on $M^{-}-\langle 1\rangle$, and then none of the $\eta_{i}^{+}$are constant there. But then all the $\eta_{i}^{+}$are exceptional for $M^{-}$. As there are more $\eta_{i}^{+}$than $\eta_{i}^{-}$, this cannot be. Thus the two families are distinct. 
7. The characters of these families are real. For, as $\theta_{i}^{-}$is 0 off $M^{-}$, its multiplicity in a character on $N\left(M^{-}\right)$depends only on the values on $M^{-}$. Since each member of $M^{-}$is conjugate to its inverse, ${O_{i}}^{-}$is real: $\eta^{-}$has real values on $M^{-}$, so that $\eta_{i}^{-}$and $\overline{\eta_{i}-}$ have the same values on ${M^{-}}^{-}$. Their restrictions to $N\left(M^{-}\right)$then contain $\theta_{i}^{-}$the same number of times. Since $(q-3 m) / 6 \geqq 3$, this means that $\eta_{i}^{-}=\overline{\eta_{i}{ }^{-}}$or $\eta_{i}^{-}$is real. Similarly $\eta_{i}^{+}$is real.

8. The remaining characters of $G$ can now be given. The members of $\left(M^{-} \cup M^{+}\right)-\langle 1\rangle$ represent $(q-3 m) / 6+(q+3 m) / 6=q / 3$ conjugate classes of $G$. The total number of conjugate classes of $G$ is then $q+8$. As $q+6$ characters have been described so far, there are two left, $\xi_{9}$ and $\xi_{10}$. Of the characters of positive 2-defect, only $\xi_{5}, \xi_{6}, \xi_{7}$, and $\xi_{8}$ are nonreal. The number of pairs of nonreal characters is the same as the number of pairs of mutually inverse classes of $G$ [12]. That number is 3 (from $T, T^{-1} ; Y T, Y T^{-1} ; J T, J T^{-1}$ ). Thus $\overline{\xi_{9}}=\xi_{10}$.

9. The rest of the chapter is devoted to determining some of the values of these new characters, including their degrees.

First the technique of paragraph I-7 and paragraph II-8 is applied to the exceptional characters. Let $V$ and $W$ stand generically for members of $M^{-}-\langle 1\rangle$ and $M^{+}-\langle 1\rangle$, respectively. $\Sigma|\chi(V)|^{2}=c(V)=q+1-3 m$, summed over the characters. Those other than the $\eta_{i}^{-}$have rational integral values on $V$; if $c$ is the contribution to the sum from these, $c \geqq 4$ (4 from the principal 2-block, from paragraph II-3). Moreover, $\eta_{i}^{-}(V)=\varepsilon^{-} \theta_{i}^{-}(V)+x, x=\eta^{-}(V)$ a rational integer. The centralizer equation in $N\left(M^{-}\right)$used to simplify the corresponding equation in $G$ gives

$$
6=q-3 m x^{2} / 6-2 \varepsilon^{-} x+c .
$$

With the restrictions given above, for $q>27$ it can only be that $x=0$ and $c=6$. When $q=27, x$ could be 0 or $\varepsilon^{-}$. If it were $\varepsilon^{-}, c$ would be 5 . Thus outside of the principal 2-block and the $\eta_{i}^{-}$, one character has value \pm 1 on $V$. As $\xi_{9}=\xi_{10}$, it is not one of these; and there are too many $\eta_{i}^{+}$(they all have the same value on $V$ ). So $x=\varepsilon^{-}$is ruled out, and again $x=0, c=6$. The same argument as for the contradiction gives $\xi_{9}(V)=\xi_{10}(V)= \pm 1$. All the other characters except the $\eta_{i}{ }^{-}$and the four in the principal 2-block are 0 on $V$.

Quite similarly, $\eta^{+}(W)=0, \xi_{9}(W)=\xi_{10}(W)= \pm 1$ and all other characters besides the $\eta_{i}{ }^{+}$and the four in the principal 2-block are 0 on $W$.

10. The $\eta_{i}^{-}$, the $\eta_{i}{ }^{+}, \xi_{9}$, and $\xi_{10}$ are of 2-defect 0 and vanish on $\langle R\rangle-\langle 1\rangle$ and $\langle S\rangle-\langle 1\rangle$ (paragraph I-7 and paragraph II-8). The congruences obtained from $\langle R\rangle$ and $\langle S\rangle$ imply that their degrees (being divisible by 8 ) are divisible by $q^{2}-1$. Since $\eta_{i}^{-}$is 0 on $M^{+}-\langle 1\rangle$ and $\eta_{i}{ }^{+}$is 0 on $M^{-}-\langle 1\rangle$, the degree of $\eta_{i}^{-}$is divisible by $q+1+3 m$ and that of $\eta_{i}^{+}$by $q+1-3 m$. Thus let $\xi_{9}(1)=\xi_{10}(1)=c\left(q^{2}-1\right)$, $\eta_{i}^{-}(1)=a\left(q^{2}-1\right)(q+1+3 m), \eta_{i}^{+}(1)=b\left(q^{2}-1\right)(q+1-3 m)$. As the sum of the squares of the degrees of the characters is $g$, we have (removing $q^{2}-1$ ) 


$$
\begin{aligned}
(q-3 m)(q+1 & +3 m)^{2}\left(a^{2}-1\right) / 6 \\
& +(q+3 m)(q+1-3 m)^{2}\left(b^{2}-1\right) / 6+2 c^{2}=2 q / 3 .
\end{aligned}
$$

Because the coefficients of $a^{2}-1$ and $b^{2}-1$ exceed $2 q / 3, a=b=1$. Then $c=m$. Thus $\eta_{i}^{-}(1)=\left(q^{2}-1\right)(q+1+3 m), \eta_{i}^{+}(1)=\left(q^{2}-1\right)(q+1-3 m)$, and $\xi_{9}(1)=\xi_{10}(1)=m\left(q^{2}-1\right)$.

Use of the congruences obtained from $\xi_{9}$ and $\xi_{10}$ restricted to $M^{-}$and $\mathrm{M}^{+}$ gives $\xi_{9}(V)=\xi_{10}(V)=-1$ and $\xi_{9}(W)=\xi_{10}(W)=1$. The orthogonality relations for $V$ and 1 give $\varepsilon^{-}=-1$; for $W$ and $1, \varepsilon^{+}=-1$.

\section{Chapter V. Completion of the Character Table}

The gaps in the table are all for elements of the 3-Sylow subgroup, and they are almost all filled from the orthogonality relations. We shall use the phrase " $A$ to $B$ " to mean the orthogonality relations applied to the classes of $A$ and $B$. A similar phrase is used for characters.

$Y$ to $S$ gives $b(Y)=-1$ and $Y T$ to $S$ gives $b(Y T)=-1$ (cf. paragraph III-1) so that $b\left(Y T^{-1}\right)=-1$ also. $V$ to $Y, W$ to $Y$, and the centralizer relation for $Y$ give $\xi_{9}(Y)=\xi_{10}(Y)=-m, \eta_{i}^{-}(Y)=\eta_{i}^{+}(Y)=-1 . V$ to $X, W$ to $X$, and the centralizer relation on $X$ give $\xi_{9}(X)=\xi_{10}(X)=-m, \eta_{i}^{-}(X)=-(q+1+3 m), \eta_{i}^{+}(X)=-(q+1-3 m)$.

$V$ to $T, W$ to $T$, and $Y$ to $T$ give $-m / 2$ for the real part of $\xi_{5}(T)$ and a linear relation connecting the real part of $\xi_{9}(T), \eta_{i}{ }^{-}(T)$, and $\eta_{i}{ }^{+}(T)$. Congruences from restriction to $F$ and the bound provided by $c(T)=2 q^{2}$, along with the fact that from $\eta_{i}^{-}(J T)=\eta_{i}^{+}(J T)=0$, it follows that $\eta_{i}^{-}(T)$ and $\eta_{i}^{+}(T)$ must be even, yield $\eta_{i}^{-}(T)=-3 m-1, \eta_{i}^{+}(T)=3 m-1$. $T$ to $T^{-1}$ gives a quadratic relation between the imaginary parts of $\xi_{5}(T)$ and $\xi_{9}(T)$. Finally, $(T, T ; J)=0$. For if $T_{1}$ and $T_{2}$ are conjugate to $T$, then $T_{1} T_{2}=J$ implies $\left(T_{1} J\right)^{-1} T_{2}\left(T_{1} J\right)=T_{1}^{-1}$, an impossibility. This gives the imaginary parts up to a sign. The fact that $\xi_{5}(T)-\xi_{5}(J T)$ belongs to a prime ideal divisor of 2 in the field of $g$ th roots of unity determines one sign, and as $\xi_{9}$ and $\xi_{10}$ have not heretofore been distinguished, the other can be chosen arbitrarily. The remaining values come from conjugation and orthogonality with the decomposition numbers.

Finally the values for $Y T: \eta_{r}$ to $\eta_{i}^{-}$gives $\eta_{i}^{-}(Y T)=\eta_{i}^{-}\left(Y T^{-1}\right)=-1$, and $\eta_{r}$ to $\eta_{i}^{+}$gives $\eta_{i}^{+}(Y T)=\eta_{i}^{+}\left(Y T^{-1}\right)=-1$. $\xi_{9}$ to $\eta_{r}$ gives $m / 2$ as the real part of $\xi_{9}(Y T)$, and $Y$ to $Y T$ gives the real part of $\xi_{5}(Y T)$ as $-m / 2$. $T$ to $Y T$ gives the sum of the imaginary parts of $\xi_{5}(Y T)$ and $\xi_{9}(Y T)$ to be 0 . Finally, the centralizer relation on $Y T$ gives these imaginary parts up to a sign. From paragraph III-12, we may replace $Y$ by a conjugate $Y_{1}$ in such a way that $Y T^{-1}$ is conjugate to $Y_{1} T$. This option allows the sign of the imaginary part of $\xi_{5}(Y T)$ to be chosen arbitrarily.

The simplicity of $G$ follows from the fact that no character takes on a value equal to its degree on any nonidentity element.

In the character table below, a listing of a paragraph number refers to exceptional values and where they are discussed (or introduced). 


\begin{tabular}{l|crrrrrr} 
Value on & 1 & $R^{a} \neq 1$ & $S^{a} \neq 1$ & $V$ & $W$ & $X$ & $Y$ \\
\hline $\begin{array}{c}\text { Character } \\
\xi_{1}\end{array}$ & 1 & 1 & 1 & 1 & 1 & 1 & 1 \\
$\xi_{2}$ & $q^{2}-q+1$ & 1 & 3 & 0 & 0 & $1-q$ & 1 \\
$\xi_{3}$ & $q^{3}$ & 1 & -1 & -1 & -1 & 0 & 0 \\
$\xi_{4}$ & $q\left(q^{2}-q+1\right)$ & 1 & -3 & 0 & 0 & $q$ & 0 \\
$\xi_{5}$ & $(q-1) m(q+1+3 m) / 2$ & 0 & 1 & -1 & 0 & $-(q+m) / 2$ & $m$ \\
$\xi_{6}$ & $(q-1) m(q+1-3 m) / 2$ & 0 & -1 & 0 & 1 & $(q-m) / 2$ & $m$ \\
$\xi_{7}$ & $(q-1) m(q+1+3 m) / 2$ & 0 & 1 & -1 & 0 & $-(q+m) / 2$ & $m$ \\
$\xi_{8}$ & $(q-1) m(q+1-3 m) / 2$ & 0 & -1 & 0 & 1 & $(q-m) / 2$ & $m$ \\
$\xi_{9}$ & $m\left(q^{2}-1\right)$ & 0 & 0 & -1 & 1 & $-m$ & $-m$ \\
$\xi_{10}$ & $m\left(q^{2}-1\right)$ & 0 & 0 & -1 & 1 & $-m$ & $-m$ \\
$\eta_{r}$ & $q^{3}+1$ & $\mathrm{I}-6$ & 0 & 0 & 0 & 1 & 1 \\
$\eta_{r}^{\prime}$ & $q^{3}+1$ & $\mathrm{I}-6$ & 0 & 0 & 0 & 1 & 1 \\
$\eta_{t}$ & $(q-1)\left(q^{2}-q+1\right)$ & 0 & II-6 & 0 & 0 & $2 q-1$ & -1 \\
$\eta_{t}^{\prime}$ & $(q-1)\left(q^{2}-q+1\right)$ & 0 & II-6 & 0 & 0 & $2 q-1$ & -1 \\
$\eta_{i}^{-}$ & $\left(q^{2}-1\right)(q+1+3 m)$ & 0 & 0 & IV-5 & 0 & $-q-1-3 m$ & -1 \\
$\eta_{i}^{+}$ & $\left(q^{2}-1\right)(q+1-3 m)$ & 0 & 0 & 0 & IV-5 & $-q-1+3 m$ & -1
\end{tabular}

\begin{tabular}{c|cccc} 
Value on & $T$ & $T^{-1}$ & $Y T$ & $Y T^{-1}$ \\
\hline $\begin{array}{c}\text { Character } \\
\xi_{1}\end{array}$ & 1 & & & \\
$\xi_{2}$ & 1 & 1 & 1 & 1 \\
$\xi_{3}$ & 0 & 1 & 0 & 1 \\
$\xi_{4}$ & 0 & 0 & 0 & 0 \\
$\xi_{5}$ & $\left(-m+i m^{2} \sqrt{ } 3\right) / 2$ & $\left(-m-i m^{2} \sqrt{ } 3\right) / 2$ & $(-m-i m \sqrt{ } 3) / 2$ & $(-m+i m \sqrt{ } 3) / 2$ \\
$\xi_{6}$ & $\left(-m+i m^{2} \sqrt{ } 3\right) / 2$ & $\left(-m-i m^{2} \sqrt{ } 3\right) / 2$ & $(-m-i m \sqrt{ } 3) / 2$ & $(-m+i m \sqrt{ } 3) / 2$ \\
$\xi_{7}$ & $\left(-m-i m^{2} \sqrt{ } 3\right) / 2$ & $\left(-m+i m^{2} \sqrt{ } 3\right) / 2$ & $(-m+i m \sqrt{ }) / 2$ & $(-m-i m \sqrt{ } 3) / 2$ \\
$\xi_{8}$ & $\left(-m-i m^{2} \sqrt{ } 3\right) / 2$ & $\left(-m+i m^{2} \sqrt{ } 3\right) / 2$ & $(-m+i m \sqrt{ } 3) / 2$ & $(-m-i m \sqrt{ } 3) / 2$ \\
$\xi_{9}$ & $-m+i m^{2} \sqrt{ } 3$ & $-m-i m^{2} \sqrt{ } 3$ & $(m+i m \sqrt{ } 3) / 2$ & $(m-i m \sqrt{ } 3) / 2$ \\
$\xi_{10}$ & $-m-i m^{2} \sqrt{ } 3$ & $-m+i m^{2} \sqrt{ } 3$ & $(m-i m \sqrt{ } 3) / 2$ & $(m+i m \sqrt{ } 3) / 2$ \\
$\eta_{r}$ & 1 & 1 & 1 & 1 \\
$\eta_{r}^{\prime}$ & 1 & 1 & 1 & 1 \\
$\eta_{t}$ & -1 & -1 & -1 & -1 \\
$\eta_{t}^{\prime}$ & -1 & -1 & -1 & -1 \\
$\eta_{i}^{-}$ & $-3 m-1$ & $-3 m-1$ & -1 & -1 \\
$\eta_{i}^{+}$ & $3 m-1$ & $3 m-1$ & -1 &
\end{tabular}




\begin{tabular}{c|ccccc} 
Value on & $J T$ & $J T^{-1}$ & $J R^{a} \neq J$ & $J S^{a} \neq J$ & $J$ \\
\hline $\begin{array}{c}\text { Character } \\
\xi_{1}\end{array}$ & 1 & & & & \\
$\xi_{2}$ & -1 & 1 & 1 & 1 & 1 \\
$\xi_{3}$ & 0 & -1 & -1 & -1 & -1 \\
$\xi_{4}$ & 0 & 0 & 1 & -1 & $q$ \\
$\xi_{5}$ & $(1-i m \sqrt{ } 3) / 2$ & $(1+i m \sqrt{ } 3) / 2$ & 0 & 1 & $-q$ \\
$\xi_{6}$ & $(-1+i m \sqrt{ } 3) / 2$ & $(-1-i m \sqrt{ } 3) / 2$ & 0 & -1 & $-(q-1) / 2$ \\
$\xi_{7}$ & $(1+i m \sqrt{ }) / 2$ & $(1-i m \sqrt{ } 3) / 2$ & 0 & 1 & $-(q-1) / 2$ \\
$\xi_{8}$ & $(-1-i m \sqrt{ } 3) / 2$ & $(-1+i m \sqrt{ } 3) / 2$ & 0 & -1 & $(q-1) / 2$ \\
$\xi_{9}$ & 0 & 0 & 0 & 0 & 0 \\
$\xi_{10}$ & 0 & 0 & 0 & 0 & 0 \\
$\eta_{r}$ & 1 & 1 & I-6 & 0 & $q+1$ \\
$\eta_{r}$ & -1 & -1 & I-6 & 0 & $-(q+1)$ \\
$\eta_{t}$ & -3 & -3 & 0 & II-6 & $3(q-1)$ \\
$\eta_{t}$ & 1 & 1 & 0 & II-6 & $-(q-1)$ \\
$\eta_{i}^{-}$ & 0 & 0 & 0 & 0 & 0 \\
$\eta_{i}^{+}$ & 0 & 0 & 0 & 0 & 0
\end{tabular}

\section{BIBLIOGRAPHY}

1. R. Brauer, On blocks of characters of groups of finite order. II, Proc. Nat. Acad. Sci. U.S.A. 32 (1946), 215-219.

2. - Zur Darstellungstheorie der Gruppen endlicher Ordnung, Math. Z. 63 (1956), 406-444.

3. — Zur Darstellungstheorie der Gruppen endlicher Ordnung. II, Math. Z. 72 (1959), 25-46.

4. - On blocks of representations of finite groups, Proc. Nat. Acad. Sci. U.S.A. 47 (1961), 1888-1890.

5. - Investigations on groups of even order. I, Proc. Nat. Acad. Sci. U.S.A., 47 (1961), 1891-1893.

6. - Exceptional characters, Seminar on Finite Groups, Harvard University, Cambridge, Mass., 1960-1961; pp. 41-45.

7. - Some applications of the theory of blocks of characters of finite groups. I, J. Algebra 1 (1964), 152-167.

8. R. Brauer and W. Feit, On the number of irreducible characters of finite groups in a given block, Proc. Nat. Acad. Sci. U.S.A. 45 (1959), 361-365.

9. W. Burnside, Theory of groups of finite order, 2nd. ed., Cambridge Univ. Press, New York, 1911.

10. C. W. Curtis and I. Reiner, Representation theory of finite groups and associative algebras, Interscience, New York, 1962.

11. L. E. Dickson, Linear groups, with an exposition of the Galois field theory, Leipzig, 1901 (reprinted: Dover, New York, 1958).

12. G. Frobenius and I. Schur, Ueber die Darstellungen der endlichen Gruppen. S.-B. Berlin Math. Ges. (1906), 186-208. 
13. M. Hall, The theory of groups, Macmillan, New York, 1959.

14. H. E. Jordan, Group-characters of various types of linear groups, Amer. J. Math. 29, (1907), 387-405.

15. G. W. Mackey, The theory of group representations. Univ. of Chicago, Chicago Ill., (mimeographed notes), 1955.

16. R. Ree, A family of simple groups associated with the simple Lie algebra of type $\left(G_{2}\right)$. Bull. Amer. Math. Soc. 66 (1960), 508-510.

17. - A family of simple groups associated with the simple Lie algebra of type $\left(G_{2}\right)$, Amer. J. Math. 83 (1961), 432-462.

18. - - Sur une famille de groupes de permutations doublement transitifs, Canad. J. Math. 16 (1964), 797-820.

19. M. Suzuki, On finite groups with cyclic Sylow subgroups for all odd primes, Amer. J. Math. 77 (1955), 657-691.

20. —- On a class of doubly transitive groups. II, Ann. of Math. 79 (1964), 514-589.

21. H. Zassenhaus, The theory of groups, 2nd. ed., Chelsea, New York, 1958.

BROWN UNIVERSITY,

Providence, RHode IsLAND 